\title{
GLOBAL BIODIVERSITY AND GEOGRAPHICAL DISTRIBUTION OF DIAPAUSING AQUATIC INVERTEBRATES: THE CASE OF THE COSMOPOLITAN BRINE SHRIMP, ARTEMIA (BRANCHIOPODA, ANOSTRACA)
}

\author{
BY \\ J. MUÑOZ ${ }^{1,3}$ ) and F. PACIOS ${ }^{2}$ ) \\ 1) Department of Wetland Ecology, Estación Biológica de Doñana (Consejo Superior \\ de Investigaciones Científicas - CSIC), Pabellón del Perú, Av. María Luisa, s/n, \\ E-41013 Sevilla, Spain \\ 2 ) Department of Conservation Biology, Estación Biológica de Doñana (Consejo Superior \\ de Investigaciones Científicas - CSIC), Pabellón del Perú, Av. María Luisa, s/n, \\ E-41013 Sevilla, Spain
}

\begin{abstract}
The genus Artemia comprises passively dispersed anostracan species with a distribution all around the world, except in Antarctica. We used both published and personal data to assess and update existing knowledge on the diversity and distribution of Artemia, in particular compiling also genetic and geographic information. Our results indicate there are three Artemia complexes, A. franciscana, A. tibetiana and A. salina, suggesting at least three undescribed, and one unidentified to date, highly isolated lineages, to be re-evaluated taxonomically. Additionally, at a global scale, our data set shows two large, poorly explored geographic regions in Central East Asia, which in future studies could provide interesting information on geographic speciation, the origin of parthenogenesis, and range expansion in this group. We also discuss the implications for conservation as derived from knowledge on the biodiversity (native and invasive species) and geographic distribution (i.e., identification of species/lineages, and regions occupied), which have major relevance for conservation management at the level of wetland ecosystems.
\end{abstract}

\section{RESUMEN}

El género Artemia está constituido por anostrácodos dispersados de forma pasiva con una distribución alrededor de todo el mundo, excepto en la Antártica. Utilizamos tanto datos propios como publicados para investigar y actualizar la información existente sobre la diversidad y distribución de Artemia, en concreto compilamos datos genéticos y geográficos. Nuestros resultados indican que existen tres complejos de Artemia, A. franciscana, A. tibetiana y A. salina, sugiriendo al menos tres linajes aislados no determinados, y uno no descrito hasta ahora, para ser re-evaluados taxonómicamente. Así mismo, y a una escala global, nuestros datos muestran dos grandes regiones geográficas

${ }^{3}$ ) Corresponding author; e-mail: quini@ebd.csic.es; Fax: +34 954621125

(C) Koninklijke Brill NV, Leiden, 2010

Crustaceana 83 (4): 465-480

Also available online: www.brill.nl/cr DOI: $10.1163 / 001121610 X 489449$ 
pobremente exploradas en el Este Central de Asia, que en futuros estudios podrían proporcionar una información interesante sobre la especiación geográfica, el origen de la partenogénesis y la expansión de rango en este grupo. También discutimos las implicaciones para la conservación derivadas del conocimiento de la biodiversidad (especies nativas e invasoras) y la distribución geográfica (i.e., identificación de especies/linajes y regiones que ocupan), lo cual tiene una gran relevancia para el manejo de la conservación a nivel de ecosistemas de humedales.

\section{INTRODUCTION}

Recording global biodiversity, in terms of the number of species, represents one of the main challenges for biologists. That challenge, however, becomes utopian because of, e.g., differences in collecting activities, like lower efforts in as yet unexplored areas (Sánchez-Fernández et al., 2008), the complications in identifying 'sibling/cryptic' species (Weaver et al., 2008), etc. Nonetheless, efforts have been made during the last decades to record global diversity and to compile biogeographical information (Edwards, 2004; Wheeler, 2004).

Also, the role of human-mediated dispersal in increasing the geographical distribution range of some species, and in enhancing the chances of invasion events is well known (Suarez \& Tsutsui, 2008). Because of the ecological impact invasions may have, species identification and knowledge of their geographical distribution, of both native and invasive taxa, constitute essential issues in ecology today.

The use of a DNA 'barcode', i.e., an inventory of DNA sequences from the standardized genomic region of the mitochondrial gene for cytochrome $c$ oxidase subunit I - COI (Hebert et al., 2003, but see also the Consortium for Barcode Life Website: http://www.barcoding.si.edu/), has been proposed to assign an unknown sample to a known species, and/or to detect previously unsampled species as distinct, assisting traditional identification methods. Though this has allowed the discovery of new species in several taxa, it has also been criticized and it currently is considered a controversial method, but well supported in general (Meyer \& Paulay, 2005; Strugnell \& Lindgren, 2007; Wiemers \& Fiedler, 2007). The use of the COI mitochondrial gene has only been tested in a few taxa of the Crustacea (cf. DeWaard et al., 2006; Costa et al., 2007; Muñoz et al., 2008).

Among crustaceans, the brine shrimp, Artemia (Branchiopoda, Anostraca) is a well-known, extremely halophilic organism with a worldwide distribution. The genus has been quite well described taxonomically, and is composed of a few species with sexual reproduction and some parthenogenetic lineages. The species inhabit saline and hypersaline lakes and wetlands, for a large part related to human activities (i.e., saltworks) for several millennia, which implies that the current distribution of various Artemia species could be derived from deliberate or accidental inoculation. 
The actual aim of our present study was to investigate if detailed knowledge on the taxonomic status and geographic distribution of the species and lineages of Artemia could be of use in (a) evaluating the state of our knowledge on the biodiversity of the genus on saline and hypersaline wetland ecosystems on a global scale; (b) identifying possibly existing relevant gaps in our knowledge on the geographic distribution in Artemia; and possibly; (c) deriving measures that could be instrumental in a proper management of the wetlands under concern.

Specifically, we focus on the following questions: (1) Does the taxonomy of Artemia need re-evaluation in the light of molecular data? (2) Does the updated, known geographical distribution of Artemia show any relevant gap of information that should be filled as soon as possible?

\section{MATERIAL AND METHODS}

Study organism

Artemia spp. have rather extensively been described in several books, with regard to their toxicology, physiology, genetics, ecology, biogeography, and applications in aquaculture (Persoone et al., 1980; Sorgeloos et al., 1987; Abatzopoulos et al., 2002), to the extent that it has been labelled the 'aquatic Drosophila' due to its advantages for use as a model organism (Gajardo \& Beardmore, 2001). This genus inhabits hypersaline (45-370 g/L) patchy habitats around the world, except Antarctica (Triantaphyllidis et al., 1998). However, the persistence of endemic lineages and native Artemia species is affected by loss of their habitats and by the introduction of the American species, A. franciscana Kellogg, 1906 in Europe and on other continents (Amat et al., 2007; McMaster et al., 2007).

The genus currently comprises six sexual species: A. franciscana Kellogg, 1906; A. persimilis Piccinelli \& Prosdocimi, 1968; A. salina (Linnaeus, 1758); A. urmiana Gunther, 1900; A. sinica Cai, 1989; and A. tibetiana Abatzopoulos, Zhang \& Sorgeloos, 1998; as well as several A. parthenogenetica Bowen \& Sterling, 1978 lineages (only present in the Old World and Australia) with different ploidy (2n, 3n, $4 n, 5 n)$. Additionally, several genetically differentiated lineages have recently been uncovered, suggesting a taxonomic re-evaluation of the genus might be required (Hou et al., 2006; Tizol-Correa, 2006; Muñoz et al., 2008).

\section{Genetic data and analyses}

We used nine haplotypes (i.e., different sequences) of a fragment of the mitochondrial COI gene from six sexual Artemia species and one diploid parthenogenetic lineage. Artemia COI sequences were downloaded from GenBank and 
aligned ( 576 base pairs) using the Sequencher ${ }^{\mathrm{TM}}$ v 4.5 software (Gene Codes Corp.) in order to assess their interspecific phylogenetic relationships. We also added a new sequence, obtained in our laboratory, from Cape Verde (see table I for details). We focused on two different objectives: (1) to investigate the phylogenetic relationships of the various Artemia species including all available COI data to shed light on the true biodiversity of the group; and (2) to explore the usefulness of the COI gene as a DNA 'barcode' in Artemia.

The evolutionary history among the Artemia sequences was inferred using the Neighbor-Joining method. Phylogenetic analyses were conducted in MEGA4 (Tamura et al., 2007) using evolutionary distances computed with the Maximum Composite Likelihood method and the Tamura-Nei substitution model. The robustness of the branches was assessed with 2000 bootstrap pseudo-replicates.

\section{Geographic data}

Localities were recorded from both published (33 references in total) and personal data (see Supplementary Information: Suppl. Inf., in the online version of this paper, at: www.brill.nl/cr). The locality coordinates were obtained through the Alexandria Digital Library Gazetteer services (see http://clients.alexandria.ucsb. edu/globetrotter/), by introducing the site name and checking the results with images of Google Earth to assess the presence of water bodies. Subsequently, the locations with their correct coordinates were entered into Excel tables, which were subsequently imported by ArcGis Desktop package (Quantum GIS Development Team, 2009). Spatial data obtained through this procedure were used to map the distribution of Artemia species around the world by creating an independent layer for each species (specific maps not included in this paper, but available on request).

Our present study includes only those maps where undescribed Artemia and A. franciscana occur, in order to visualize the overlapping between unknown or invasive species and the main 'hotspots' identified on Earth (i.e., geographic areas characterized both by containing a large number of endemic species and having serious levels of habitat loss; see Website: http://www.biodiversityhotspots.org/Pages/ default.aspx).

Van Stappen (2002) listed the most recent inventory of localities where Artemia can be found, which included previously described sites and those obtained through personal and informal communication, correspondence, or trip reports (see Van Stappen, 2002 for details). Here, we removed those sites reported only through personal communication if they could not be localized geographically with the method described above. The present data also correct geographical coordinates that were incorrectly stated in previous reports (see Suppl. Inf. for details). 


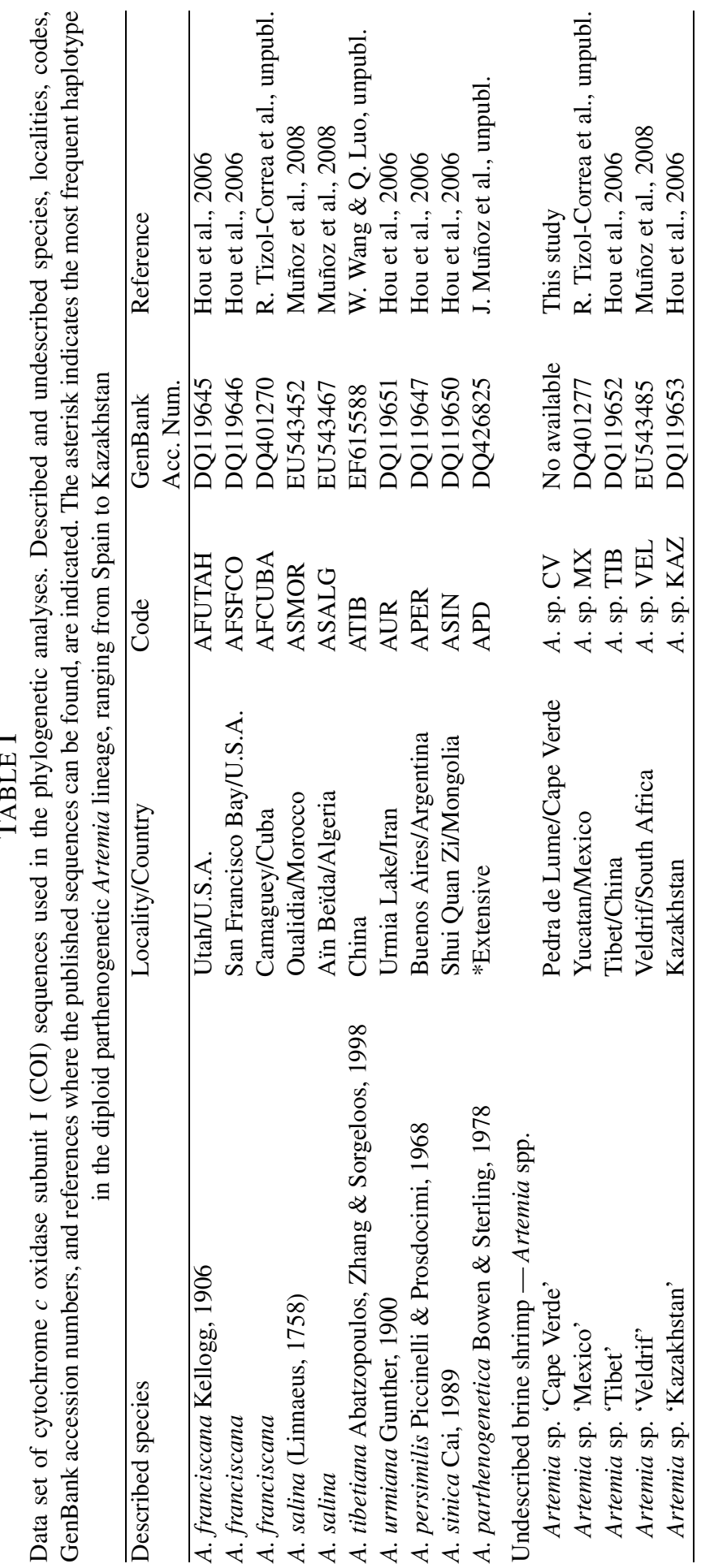


RESULTS

Phylogenetic relationships

Our phylogenetic reconstruction using COI (fig. 1) is congruent with the pattern of evolutionary relationships reported previously for Artemia (see Muñoz et al., 2008 and references therein). Similar results were found with other molecular markers, such as the nuclear Internal Transcribed Spacer 1 (ITS1) or the mitochondrial 16S rDNA unit (Baxevanis et al., 2006; Hou et al., 2006). However, with the addition of all available sequences, our results show the presence of three isolated Artemia complexes with one highly isolated lineage within each one: the $A$. franciscana complex, A. tibetiana complex, and A. salina complex. The A. franciscana and A. salina complex are both monophyletic, while the $A$. tibetiana complex shows an undescribed lineage (A. sp. TIB) as a paraphyletic unit. The A. tibetiana species cluster, in their turn, in a monophyletic way with the parthenogen and other Central Asian species (AUR; A. sp. KAZ).

In addition, our results show the presence of three undescribed and highly isolated Artemia lineages (A. sp. CV from Cape Verde; A. sp. TIB from Tibet; A. sp. KAZ from Kazakhstan), and one unidentified lineage (ASVEL from Veldrif - South Africa).

\section{Geographic distribution}

The Artemia data here presented show our lack of knowledge on biodiversity (undescribed and unidentified species), and subsequently on biogeographical information (distribution range), in a large part of the world: Africa, Europe, Asia, Australia, and South America (see fig. 2a, b). This information shortage includes almost all American hotspots (fig. 2b), such as the Caribbean, the California coast, Mesoamerica or Western Ecuador, and five hotspots in the rest of the continents (fig. 2a).

Additionally, our results indicate that non-hotspot areas are in need of more scientific attention through further study. Two large regions, in Central and CentralEast Asia could be categorized as 'unknown areas', comprising a high number of sites where Artemia occurs, but where species invariably remain undescribed currently. From the total of our listed geo-referenced localities where Artemia can been found $(\mathrm{N}=499 ; 78$ in North America, 92 in Africa, 153 in Asia, 124 in the Caribbean, Central \& South America, 172 in Europe, and 14 in Australia \& New Zealand; see details in Suppl. Inf., in the online version of this paper, at: www.brill.nl/cr), the number of localities with undescribed species ranges from 6 to 111 (see table II). Asia shows 111 localities, while the region of Australia and New Zealand shows six. However, the percentage of localities with undescribed 


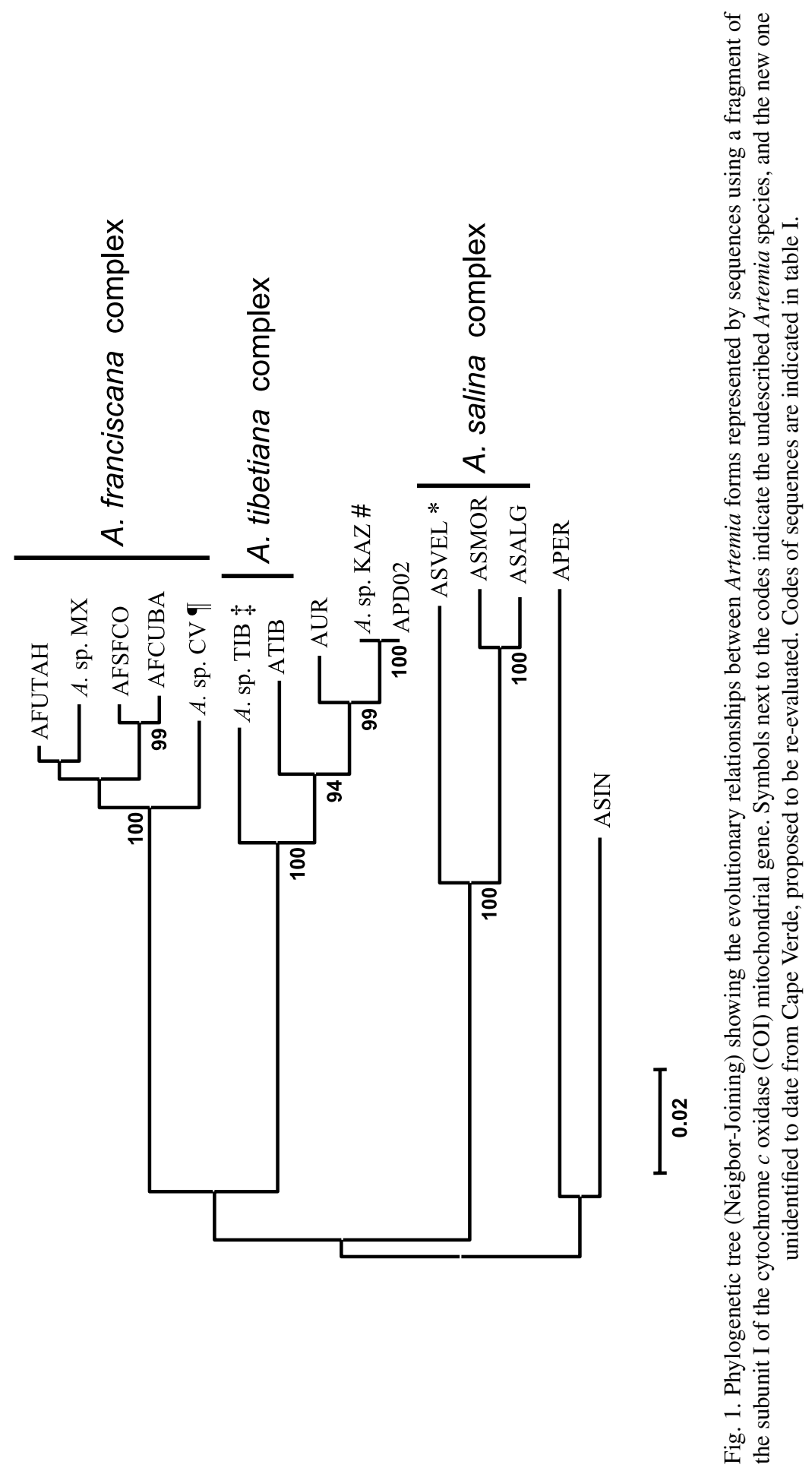




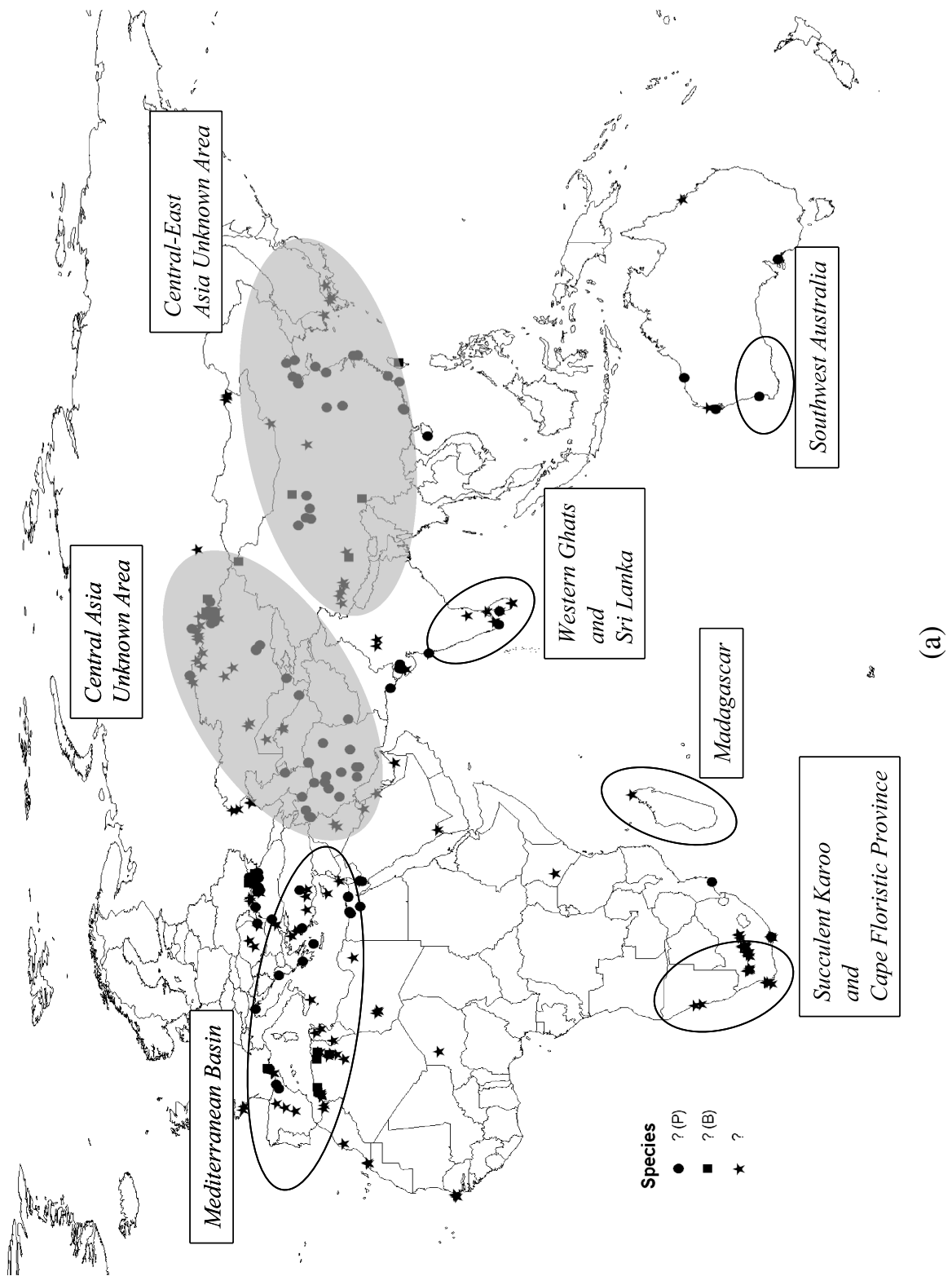




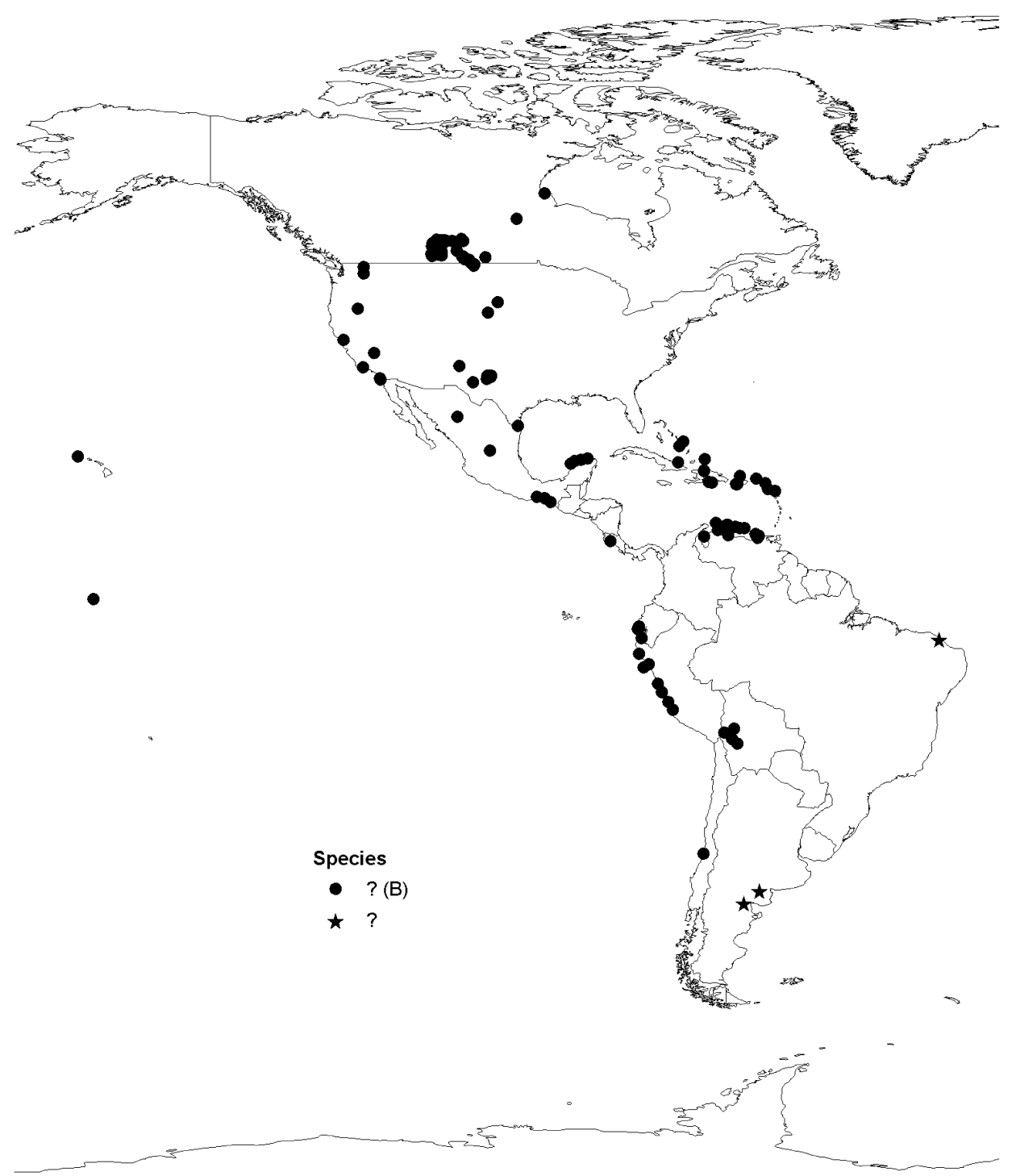

(b)

Fig. 2. a, Distribution of undescribed Artemia in Europe, Africa, Asia, and Australia; stars correspond to localities holding Artemia with unknown reproductive mode (?); squares correspond to localities holding sexual Artemia (?(B)); circles correspond to localities holding parthenogenetic Artemia (?(P)); open circles correspond to those hotspots described by Myers et al. (2000) where undescribed Artemia has been recorded; grey circles correspond to those large regions where a substantial lack of information exists on Artemia biodiversity; b, distribution of undescribed Artemia in America; stars correspond to localities holding Artemia with unknown reproductive mode (?); circles filled correspond to localities holding sexual Artemia (?(B)). 


\section{TABLE II}

Number of localities with undescribed Artemia species all around the world. ?(B) $=$ undescribed sexual Artemia; ?(P) = undescribed parthenogenetic Artemia; ? = undescribed Artemia and unknown reproductive mode. The percentage values of localities with undescribed species were calculated taking into account the total number of localities (described and undescribed; $N=499$ ) as is listed in Suppl. Inf. on Website www.brill.nl/cr

\begin{tabular}{lrrrcc}
\hline Country/area & $?(\mathrm{~B})$ & $?(\mathrm{P})$ & $?$ & $\begin{array}{c}\text { Total } \\
\text { localities }\end{array}$ & $\begin{array}{c}\text { \% localities with } \\
\text { undescribed species }\end{array}$ \\
\hline North America & 58 & 0 & 0 & 78 & 78.4 \\
Africa & 5 & 8 & 57 & 92 & 76.0 \\
Asia & 5 & 59 & 47 & 153 & 72.5 \\
Caribbean, Central \& South America & 59 & 0 & 3 & 124 & 50.0 \\
Europe & 9 & 35 & 35 & 172 & 46.0 \\
Australia \& New Zealand & 0 & 4 & 2 & 14 & 42.9 \\
\hline
\end{tabular}

Artemia is higher in North America (78.4\%). The lowest percentage was found in Australia and New Zealand (42.9\%).

Finally, our data set indicates an important overlap between the areas currently invaded by A. franciscana and several hotspots (see Supplementary Map: Suppl. Map, in the online version of this paper, at: www.brill.nl/cr), which could be interesting in future studies on saline and hypersaline wetland conservation issues.

\section{DISCUSSION}

\section{Taxonomic re-evaluation}

Though genetic studies have revealed a few candidate cryptic species in intensively studied vertebrate groups (Hebert et al., 2004a), the potential for detecting new species is much higher in invertebrates (e.g., Gómez et al., 2002; Hebert et al., 2004b), but these are as yet understudied. It is well known, that the number of species as well as the taxonomy of aquatic invertebrates currently are insufficiently clear, and far from reality (IUCN, 2004; Martens et al., 2008). Additionally, recent studies suggest that geographic speciation in small invertebrates is a commoner phenomenon than in large invertebrates and in vertebrates, especially in those with worldwide distribution (Mills et al., 2007), such as aquatic invertebrates.

Combining the above data with our results, we propose three Artemia complexes, and suggest four Artemia lineages to be re-evaluated (see fig. 1). This work represents the first approach that analyses all available COI sequences in Artemia (geographically and phylogenetically representative) and identifies, at a global scale, new highly isolated lineages using the molecular marker COI as a DNA 'barcode' tool. 
Within the proposed A. salina complex, the genetic divergence among the Mediterranean A. salina populations and the South African A. salina population suggests that the lineage ASVEL (symbol * in fig. 1) could be a different sexual species, as has recently been discussed by Muñoz et al. (2008). This finding has major implications for the conservation status of Anostraca in the Western Cape, South Africa, where A. salina is not considered endemic (De Roeck et al., 2007). On the other hand, within the proposed A. tibetiana complex, a situation similar to that in the A. salina complex is found. Hou et al. (2006) identified a not yet formally described sexual lineage $A$. sp. TIB (symbol $\ddagger$ in fig. 1), but it was poorly discussed. They concluded that the Tibetan Artemia belongs to A. urmiana. However, our analyses indicate that a differentiated lineage exists in Tibet (ATIB - cf. W. Wang \& Q. Luo, unpubl. data) suggesting a paraphyletic relationship in the A. tibetiana lineages.

Additionally, the sexual Artemia species from Kazakhstan (Pilla \& Beardmore, 1994) (symbol \# in fig. 1), which clusters with a parthenogen and Central Asian Artemia (A. urmiana, A. tibetiana), remains taxonomically undescribed to date (Hou et al., 2006; Muñoz et al., 2008). Specifically, A. sp. KAZ shows COI sequences identical to those of diploid parthenogens (J. Muñoz, unpubl. data) suggesting that, although a few authors have used it, either a possible identification error has occurred, or this undescribed sexual species is the likely diploid ancestor of the parthenogen.

The most relevant and surprising find in our survey, is the presence of a highly differentiated lineage identified as A. franciscana from Cape Verde (A. sp. CV symbol II in fig. 1). Tizol-Correa (2006) described a relatively high genetic isolation in Central American populations (Cuba and Mexico) with respect to North American populations (see also Muñoz et al., 2008). However, Cape Verde is out of the native distribution range of $A$. franciscana. We could hypothesize that population originated through deliberate commercial inoculations in a similar way as it has invaded Europe and other continents (Amat et al., 2007; Muñoz et al., 2009). Nevertheless, the genetic divergence of the lineage from Cape Verde when compared to other American populations is much higher than that of any other haplotype of A. franciscana from the invaded areas (Spain, Portugal, France, Morocco, Italy - J. Muñoz, unpubl. data). An alternative hypothesis is an introduction from a South American population (e.g., Chile, which shows high genetic divergence when compared to other American populations through allozyme analysis; Gajardo et al., 1995), but unfortunately there are no COI data available from South America to test that hypothesis. Another possibility, non-investigated to date, is that the original geographic distribution range of A. franciscana was not exclusively America. This could explain the close phylogenetic relationships among $A$. franciscana and Asian Artemia species (A. sinica, A. tibetiana, and A. urmiana), and some parthenogenetic lineages (Baxevanis et al., 2006). 


\section{Lack of biogeographic information}

Although substantial efforts through field trips and expeditions show the presence of Artemia throughout the world, our current knowledge of the biodiversity and subsequent geographic distribution of units of the genus is only limited. Our updated biogeographic data set provides, for the first time, the maps of all the identified and undescribed Artemia species around the world (see Suppl. Inf., in the online version of this paper, at: www.brill.nl/cr).

To avoid a large number of maps in this paper, we include those really relevant to show the shortage of species identification over extensive geographic areas (fig. 2a, b; specific maps can be provided on request). However, not only the information on the biodiversity and the distribution of the species are important for ecology, speciation, and conservation. Biological invasion, especially in those areas characterized both by containing large numbers of endemic species and having serious levels of habitat loss (i.e., hotspots), constitute one of the main causes of species extinction (local or general) in nature (Clavero \& García-Berthou, 2005). Therefore, we also include the map that shows the spread of the invasive species, A. franciscana, in the rest of the world (see Suppl. Map, in the online version of this paper, at: www.brill.nl/cr).

The origin of parthenogenesis in Artemia has been supposed to be located in the Mediterranean Basin. However, recent evidence shows that this location can be wrong, and that it can be referred to Central Asia (J. Muñoz, unpubl. data). The close phylogenetic relationships among the sexual Artemia from Kazakhstan and other Asian Artemia species (A. urmiana, A. tibetiana) support this view. Additionally, Asia is the geographic area in the world where a higher number of parthenogenetic populations is present (see table II and fig. 2a). As in other studies at a global scale (Mills et al., 2007), research focused on Central and CentralEast Asia might well uncover new lineages, helping to understand the evolutionary aspects of the asexual reproduction in this group.

\section{Implications for conservation}

Thirteen years ago, New (1995) published the first book considering invertebrates in conservation biology at a global scale. Wetlands (i.e., worldwide aquatic ecosystems) are considered for conservation by the Ramsar Convention (Convention on Wetlands, 1971) if they support endangered species of vertebrates or the presence of large plant communities. On the other hand, Belk (1998: 149) stated: "In practical terms, habitat is often conserved as a result of its importance to some species or group of species that have become the focus of human concern". However, in many cases species exploited by humans are not conservation targets, and one example of that is Artemia. 
Artemia franciscana is currently considered an invasive species in hypersaline aquatic habitats around the world, mainly introduced for the aquaculture business (Amat et al., 2007). In the last 40 years, it has been recorded in Australia, China, Iran, Japan, Kenya, Madagascar, the Mediterranean Basin, New Zealand, and Vietnam (Muñoz et al., 2009; Ruebhart et al., 2008). Our results show an important coincidence between the areas currently invaded by this anostracan and several hotspots described by Myers et al. (2000) (see Suppl. Map, in the online version of this paper, at: www.brill.nl/cr). Nevertheless, we want to emphasize those regions not to be recognized as hotspots, where the presence of Artemia is known, but specific identification is still lacking (and consequently their uncertain geographic distribution) (fig. 2a). The presence of the invasive A. franciscana in the Old World implies both local and/or regional extinction of autochthonous Artemia species (Amat et al., 2007) and, thus, the inherent disequilibrium of the invaded aquatic ecosystems. It has been suggested, for instance, that the presence of $A$. franciscana could decrease the abundance of waterfowl due to the lack of parasitism affecting the invasive species, and this species being more evasive as a food source for those birds (Sánchez et al., 2009).

Sala et al. (2000) argued that ecosystems with a Mediterranean climate (including aquatic systems) will experience the greatest proportional change in biodiversity because of the substantial influence of general biotic exchange. Additionally, anthropogenic activities accelerate the extinction of species by means of the transformation and fragmentation of habitats and landscapes (see Pertoldi et al., 2007, for a review) and by the introduction of allochthonous species (see Mooney \& Hobbs, 2000, for a review). In ways similar to A. franciscana, used in aquaculture (Amat et al., 2007), other aquatic invertebrates such as Daphnia lumholtzi G. O. Sars, 1885 have spread via human intervention (Havel et al., 2000). Mergeay et al. (2005) identified the presence of a single American clone of Daphnia pulex Leydig, 1860 in Kenia, likely introduced accidentally by humans, which has become to dominate this region as an invasive lineage.

Finally, the incorrect identification of species in regions with high biodiversity, and the spatial scale at which wetlands are surveyed (Angeler et al., 2008), can lead to confusing management and conservation strategies. For example, A. salina has been described in South Africa in a survey about the status of conservation of wetlands in this area (De Roeck et al., 2007), but as Muñoz et al. (2008) reported, and the present study shows, the Artemia population recorded from South Africa should be considered a new species, probably endemic to that region. Therefore, discoveries like these will invariably imply the reinforcement of conservation measures, most of them involving protection of the wetland habitat at issue, as a whole. 


\section{ACKNOWLEDGEMENTS}

We are grateful to Prof. F. Amat from Instituto de Acuicultura Torre de la Sal - CSIC (Castellón, Spain) for help with the geographical coordinates of Artemia localities. Dr. Jordi Figuerola and Dr. Iván Gómez from Estación Biológica de Doñana - CSIC (Sevilla, Spain) provided constructive criticism on an earlier version of the manuscript. We are also indebted to Dr. J. C. von Vaupel, Managing Editor of Crustaceana (Leiden, Netherlands), for his invaluable job, detailed comments, and suggestions on an earlier version of this paper. This study was funded by the Spanish Ministerio de Educación y Ciencia (projects BOS200302486 and CGL 2006-05085 BOS).

\section{REFERENCES}

Abatzopoulos, T. J., J. A. Beardmore, J. S. Clegg \& P. Sorgeloos (eds.), 2002. Artemia: basic and applied biology: 1-286. (Kluwer Academic Publishers, Dordrecht).

Amat, F., F. Hontoria, J. C. NAVArro, N. Vieira \& G. Mura, 2007. Biodiversity loss in the genus Artemia in the western Mediterranean region. Limnetica, 26: 177-194.

Angeler, D. G., O. Viedma, S. Sanchez-Carrillo \& M. Alvarez-Cobelas, 2008. Conservation issues of temporary wetland Branchiopoda (Anostraca, Notostraca: Crustacea) in a semiarid agricultural landscape: what spatial scales are relevant? Biological Conservation, 141: 1224-1234.

Baxevanis, A. D., I. Kappas \& T. J. Abatzopoulos, 2006. Molecular phylogenetics and asexuality in the brine shrimp Artemia. Molecular Phylogenetics and Evolution, 40: 724-738.

BELK, D., 1998. Global status and trends in ephemeral pool invertebrate conservation: implications for Californian fairy shrimp. In: C. W. Witham, E. T. Bauder, D. Belk, W. R. FERREN, JR. \& R. ORNDUFF (eds.), Ecology, conservation, and management of vernal pool ecosystems - Proceedings from a 1996 conference: 147-150. (California Native Plant Society, Sacramento, CA).

Clavero, M. \& E. García-Berthou, 2005. Invasive species are a leading cause of animal extinctions. Trends in Ecology and Evolution, 20(3): 110.

CONVENTION ON WETLANDS, 1971. Ramsar Convention on Wetlands of International Importance. Ramsar, Iran. Available on web site http://www.ramsar.org/

Costa, F. O., J. R. DeWaArd, J. Boutillier, S. Ratnasingham, R. T. Dooh, M. HAJibabaei \& P. D. N. Hebert, 2007. Biological identificacion through DNA barcodes: the case of Crustacea. Canadian Journal of Fisheries and Aquatic Sciences, 64: 272-295.

De Roeck, E. R., B. J. Vanschoenwinkel, J. A. Day, Y. XU, L. RaitT \& L. Brendonck, 2007. Conservation status of large branchiopods in the western cape, South Africa. Wetlands, 27(1): 162-173.

DeWaard, J. R., V. Sacherova, M. E. A. Cristescu, E. A. Remigio, T. J. Crease \& P. D. N. HEBERT, 2006. Probing the relationships of the branchiopod crustaceans. Molecular Phylogenetics and Evolution, 39: 491-502.

EDWARDS, J. L., 2004. Research and societal benefits of the global biodiversity information facility. BioScience, 54: 485-486.

Gajardo, G. \& J. A. BeArdmore, 2001. Coadaptation: lessons from the brine shrimp Artemia, 'the aquatic Drosophila' (Crustacea; Anostraca). Revista Chilena de Historia Natural, 74: 65-72. 
Gajardo, G., M. Da Conceicao, L. Weber \& J. A. Beardmore, 1995. Genetic variability and interpopulational differentiation of Artemia strains from South America. Hydrobiologia, 302: 21-29.

Gomez, A., G. J. Adcock, D. H. Lunt \& G. R. Carvalho, 2002. The interplay between colonization history and gene flow in passively dispersing zooplankton: microsatellite analysis of rotifer resting egg banks. Journal of Evolutionary Biology, 15: 158-171.

Havel, J. E., J. K. Colbourne \& P. D. N. Hebert, 2000. Reconstructing the history of intercontinental dispersal in Daphnia lumholtzi by use of genetic markers. Limnology and Oceanography, 45: 1414-1419.

Hebert, P. D. N., A. Cywinska, S. L. BALl \& J. R. DewaARd, 2003. Biological identifications through DNA barcodes. Proceedings of the Royal Society, (B) 270: 313-321.

Hebert, P. D. N., E. H. Penton, J. M. Burns, D. H. Janzen \& W. Hallwachs, 2004 (cf. a). Ten species in one: DNA barcoding reveals cryptic species in the neotropical skipper butterfly Astraptes fulgerator. Proceedings of the National Academy of Sciences of the USA, 101: 41.

Hebert, P. D. N., M. Y. Stoeckle, T. S. Zemlak \& C. M. Francis, 2004 (cf. b). Identification of birds through DNA barcodes. PLoS Biology, 2: e312.

Hou, L., X. BI, X. Zou, C. He, L. YANG, R. Qu \& Z. LIU, 2006. Molecular systematics of bisexual Artemia populations. Aquaculture Research, 37: 671-680.

IUCN, 2004. Red list of threatened species ${ }^{\mathrm{TM}}$ - A global assessment. J. E. M. BAILliE, C. Hilton-TAYlor \& S. N. StUART (eds.). Available at web site http://www.iucn.org/ themes/ssc/red_list_2004/GSAexecsumm_EN.htm

Martens, K., I. Schön, C. Meisch \& D. J. Horne, 2008. Global diversity of ostracods (Ostracoda, Crustacea) in freshwater. Hydrobiologia, 595: 185-193.

McMaster, K., A. Savage, T. Finston, M. S. Johnson \& B. Knott, 2007. The recent spread of Artemia parthenogenetica in Western Australia. Hydrobiologia, 576: 39-48.

Mergeay, J., D. Verschuren \& L. De Meester, 2005. Cryptic invasion and dispersal of an American Daphnia in East Africa. Limnology and Oceanography, 50: 1278-1283.

MeYer, C. P. \& G. PAUlAY, 2005. DNA barcoding: error rates based on comprehensive sampling. PLoS Biology, 3(12): e422.

Mills, S., D. H. LunT \& A. Gomez, 2007. Global isolation by distance despite strong regional phylogeography in a small metazoan. BioMed Central (BMC) Evolutionary Biology, 7: 225.

Mooney, H. A. \& R. J. HobBs, 2000. Invasive species in a changing world: 1-439. (Island Press, Washington, D.C.).

Muñoz, J., A. Gomez, A. J. Green, J. Figuerola, F. Amat \& C. Rico, 2008. Phylogeography and local endemism of the native Mediterranean brine shrimp Artemia salina (Branchiopoda: Anostraca). Molecular Ecology, 17: 3160-3177.

Muñoz, J., A. J. Green, J. Figuerola, F. Amat \& C. Rico, 2009. Characterization of polymorphic microsatellite markers in the brine shrimp Artemia (Branchiopoda, Anostraca). Molecular Ecology Resources, 9: 547-550.

Myers, N., R. A. Mittermeier, C. G. Mittermeier, G. A. B. Da Fonseca \& J. Kent, 2000. Biodiversity hotspots for conservation priorities. Nature, London, 403: 853-858.

NEW, T. R., 1995. Introduction to invertebrate conservation biology: 1-194. (Oxford University Press, Oxford).

Persoone, G., P. Sorgeloos, O. Roels \& E. Jaspers (eds.), 1980. The brine shrimp Artemia. Ecology, culturing, use in aquaculture: 1-456. (Universa Press, Wetteren).

Pertoldi, C., R. Bijlsma \& V. Loeschcke, 2007. Conservation genetics in a globally changing environment: present problems, paradoxes and future challenges. Biodiversity and Conservation, 16: 4147-4163.

Pilla, E. J. S. \& J. A. BeARDMORE, 1994. Genetic and morphometric differentiation in Old World bisexual species of Artemia (the brine shrimp). Heredity, 73: 47-56. 
Quantum GiS Development Team, 2009. GNU General Public License. Available at web site http://qgis.osgeo.org

RUEBHART, D. R., I. E. COCK \& G. R. SHAW, 2008. Invasive character of the brine shrimp Artemia franciscana Kellogg, 1906 (Branchiopoda: Anostraca) and its potential impact on Australian inland hypersaline waters. Marine and Freshwater Research, 59: 587-595.

Sala, O. E., F. S. Chapin, III, J. J. Armesto, E. Berlow, J. Bloomfield, R. Dirzo, E. Huber-Sanwald, L. F. Huenneke, R. B. Jackson, A. Kinzing, R. Leemans, D. M. Lodge, H. A. Mooney, M. Oesterheld, N. L. Poff, M. T. Sykes, B. H. WALKER, M. WALKER \& D. H. WALL, 2000. Global biodiversity scenarios for the year 2100. Science, New York, 287: 1770-1774.

Sánchez, M. I., F. Hortas, J. Figuerola \& A. J. Green, 2009. Sandpipers select red brine shrimps rich in both carotenoids and parasites. Ethology, 115: 196-200.

SÁnChez-Fernández, D., J. M. Lobo, P. Abellan, I. Ribera \& A. Millan, 2008. Bias in freshwater biodiversity sampling: the case of Iberian water beetles. Diversity and Distributions, 14(5): 754-762.

Sorgeloos, P., D. A. Bengtson, W. Decleir \& E. JAspers (eds.), 1987. Artemia research and its applications: 1-556. (Universa Press, Wetteren).

Strugnell, J. M. \& A. R. Lindgren, 2007. A barcode of life database for the Cephalopoda? Considerations and concerns. Reviews in Fish Biology and Fisheries, 17: 337-344.

SuAREZ, A. V. \& N. D. Tsutsui, 2008. The evolutionary consequences of biological invasions. Molecular Ecology, 17: 351-360.

Tamura, K., J. Dudley, M. Nei \& S. Kumar, 2007. MEGA4: Molecular Evolutionary Genetics Analysis (MEGA) software version 4.0. Molecular Biology and Evolution, 24(8): 1596-1599.

Tizol-CorreA, R. A., 2006. Contribución a la caracterización molecular, citogenética, morfométrica y bioquímica del camarón de salmuera Artemia de Cuba y Sur de México. (Ph. D. Thesis; PDF file available at: http://iodeweb1.vliz.be/odin/bitstream/1834/1546/1/Tesis\% 20Doctoral\%20R\%20tizol.pdf).

Triantaphyllidis, G. V., T. J. Abatzopoulos \& P. Sorgeloos, 1998. Review of the biogeography of the genus Artemia (Crustacea, Anostroca). Journal of Biogeography, 25: 213-226.

Van Stappen, G., 2002. Zoogeography. In: Th. J. Abatzopoulos, J. A. Beardmore, J. S. ClegG \& P. Sorgeloos (eds.), Biology of aquatic organisms. Artemia: basic and applied biology: 171-224. (Kluwer Academic Publishers, Dordrecht).

Weaver, K. F., M. Perez-Losada, R. P. Guralnick, A. Nelson, S. Blatt \& K. A. CRANDALl, 2008. Assessing the conservation status of the land snail Oreohelix peripherica wasatchensis (family Oreohelicidae). Conservation Genetics, 9: 907-916.

WHEELER, Q. D., 2004. What if GBIF? BioScience, 54: 717.

WiEMERS, M. \& K. FiedLER, 2007. Does the DNA barcoding gap exist? - a case study in blue butterflies (Lepidoptera: Lycaenidae). Frontiers in Zoology, 4: 8. 


\section{ONLINE APPENDICES}

We have listed six different areas all around the world where Artemia is present. Coordinates are given in decimal degrees. Locality indications in red are those found by authors (see Material and Methods for details) and not indicated in references. ?(B) $=$ undescribed sexual Artemia; $?(\mathrm{P})=$ undescribed parthenogenetic Artemia $; ?=$ undescribed Artemia and unknown reproductive mode. Asterisk indicates wrong coordinates in references.

\section{AFRICA}

\begin{tabular}{|c|c|c|c|c|c|}
\hline Country & Locality & $\begin{array}{l}\text { Lati- } \\
\text { tude }\end{array}$ & $\begin{array}{l}\text { Longi- } \\
\text { tude }\end{array}$ & $\begin{array}{l}\text { Species (repro- } \\
\text { ductive mode) }\end{array}$ & $\begin{array}{l}\text { Refe- } \\
\text { rence }\end{array}$ \\
\hline \multirow[t]{17}{*}{ Algeria } & Chegga Oase & 34.48 & 5.88 & ? & [16] \\
\hline & Chott Djeloud & 34.05 & 6.33 & $?$ & [16] \\
\hline & Chott Merouan & 34.00 & 6.17 & $?(\mathrm{~B})$ & [16] \\
\hline & Chott Ouargla & 31.95 & 5.33 & $?$ & [16] \\
\hline & Dayet Morselli & 35.50 & -0.77 & $?$ & [16] \\
\hline & El-Menaceria & 35.69 & -0.22 & $?$ & {$[16]$} \\
\hline & Garaet et Tarf Salt Lake & 35.67 & 7.15 & A. salina $(\mathrm{B})$ & J. Muñoz, unpubl. \\
\hline & Gharabas Lake & 35.58 & -0.42 & $?$ & {$[16]$} \\
\hline & Mellaha Guergour El-Amri & 35.98 & 5.25 & $?(\mathrm{~B})$ & {$[16]$} \\
\hline & Salin de Bethioua & 35.80 & -0.22 & $?$ & [16] \\
\hline & Sebket Djendli & 35.72 & 6.53 & $?$ & [16] \\
\hline & Sebket Ez Zemouk & 35.88 & 6.55 & $?$ & [16] \\
\hline & Sebket Oran & 35.53 & -0.80 & $?$ & [16] \\
\hline & Sebkha Azrew & 35.72 & -0.13 & $?(\mathrm{~B})$ & [16] \\
\hline & Sebkha N'zouri & 35.83 & 6.58 & $?(\mathrm{~B})$ & [16] \\
\hline & Sebkha Sidi Bouzian & 35.87 & 0.58 & $?(\mathrm{~B})$ & [16] \\
\hline & Tougourd & 33.10 & 6.12 & $?$ & {$[16]$} \\
\hline \multirow[t]{2}{*}{ Cape Verde } & Pedra de Lume, Sal Island & 16.77 & -22.88 & A. franciscana $(\mathrm{B})$ & [28] \\
\hline & Santa Maria, Sal Island & 16.55 & -22.90 & A. franciscana (B) & [28] \\
\hline \multirow[t]{8}{*}{ Egypt } & Bourg El-Arab & 30.92 & 29.53 & $?(\mathrm{P})$ & [16] \\
\hline & El Max Saline (Alexandria) & 31.12 & 29.83 & $?(\mathrm{P})$ & {$[16]$} \\
\hline & Ismailia & 30.60 & 22.25 & $?$ & [16] \\
\hline & Port Fouad & 31.25 & 32.32 & $?(\mathrm{P})$ & {$[16]$} \\
\hline & Port Said & 31.25 & 32.28 & $?(\mathrm{P})$ & [16] \\
\hline & Qarun Lake & 29.45 & 30.68 & $?(\mathrm{P})$ & [16] \\
\hline & Solar Lake (Sinai) & 29.17 & 34.83 & $?(\mathrm{P})$ & [16] \\
\hline & Wadi Natron & 30.17 & 30.45 & A. salina $(\mathrm{B})$ & {$[16]$} \\
\hline \multirow[t]{3}{*}{ Kenya } & Elmenteita & -0.45 & 36.25 & $?$ & [16] \\
\hline & Fundisha & -3.03 & 40.13 & A. salina $(\mathrm{B})$ & {$[16]$} \\
\hline & Fundisha & -3.03 & 40.13 & A. franciscana (B) & J. Muñoz, unpubl. \\
\hline
\end{tabular}




\begin{tabular}{|c|c|c|c|c|c|}
\hline Country & Locality & $\begin{array}{l}\text { Lati- } \\
\text { tude }\end{array}$ & $\begin{array}{l}\text { Longi- } \\
\text { tude }\end{array}$ & $\begin{array}{l}\text { Species (repro- } \\
\text { ductive mode) }\end{array}$ & $\begin{array}{l}\text { Refe- } \\
\text { rence }\end{array}$ \\
\hline \multirow[t]{4}{*}{ Libya } & Gabr Acun (Fezzan) & 27.00 & 13.00 & $?$ & [16] \\
\hline & Mandara & 26.67 & 13.33 & A. salina $(\mathrm{B})$ & $\begin{array}{l}\text { J. Muñoz, } \\
\text { unpubl. }\end{array}$ \\
\hline & Quem el Ma & 26.68 & 13.37 & $?$ & [16] \\
\hline & Trouna & 26.83 & 13.50 & $?$ & [16] \\
\hline \multirow[t]{3}{*}{ Madagascar } & Ankiembe Saltworks (Tulear) & -23.35 & 43.65 & A. parth. 3n $(\mathrm{P})$ & {$[26]$} \\
\hline & Ifaty Saltworks & -23.15 & 43.62 & A. franciscana $(\mathrm{B})$ & [16] \\
\hline & Salins de Diego Suarez & -12.32 & 49.28 & $?$ & [16] \\
\hline Mocambique & Lagua Quissico & -24.68 & 34.77 & $?(\mathrm{P})$ & [16] \\
\hline \multirow[t]{12}{*}{ Morocco } & Larache & 35.20 & -2.33 & A. parth. $2 \mathrm{n}(\mathrm{P})$ & {$[4] ;[16]$} \\
\hline & Larache & 35.20 & -2.33 & A. parth. $4 \mathrm{n}(\mathrm{P})$ & [4]; [16] \\
\hline & Mar Chica Lagoon & 35.10 & -2.73 & A. parth. $2 \mathrm{n}(\mathrm{P})$ & [8] \\
\hline & Mar Chica Lagoon & 35.10 & -2.73 & A. franciscana $(\mathrm{B})$ & {$[8]$} \\
\hline & Moulaya Estuary & 35.12 & -2.33 & $?$ & {$[16]$} \\
\hline & Oualidia & 32.73 & -9.02 & A. salina $(\mathrm{B})$ & {$[7]$} \\
\hline & Qued Ammafatma & 28.30 & -12.00 & $?$ & [16] \\
\hline & Qued Chebeica & 28.42 & -11.83 & $?$ & [16] \\
\hline & Sebket Bon Areg & 35.17 & -2.83 & $?$ & [16] \\
\hline & Sebket Zima & 32.08 & -8.67 & $?$ & [16] \\
\hline & Souzama and Marocaines Salterns & 32.73 & -9.02 & A. salina $(\mathrm{B})$ & [7] \\
\hline & Souzama and Marocaines Salterns & 32.73 & -9.02 & A. parth. 2n $(\mathrm{P})$ & [7] \\
\hline \multirow[t]{4}{*}{ Namibia } & Henties Bay Salt Refin. & -22.00 & 14.27 & $?$ & [16] \\
\hline & Vineta Swakopmund Saltworks & -22.67 & 14.57 & A. parth. $2 \mathrm{n}(\mathrm{P})$ & {$[16] ;[30]$} \\
\hline & Vineta Swakopmund Saltworks & -22.67 & 14.57 & A. parth. $4 \mathrm{n}(\mathrm{P})$ & {$[16] ;[30]$} \\
\hline & Walvis Bay & -22.93 & 14.50 & $?$ & [16] \\
\hline Niger & Teguidda In Tessoun & 17.43 & 6.65 & $?$ & [16] \\
\hline \multirow[t]{3}{*}{ Senegal } & Dakar & 14.57 & -17.48 & $?$ & {$[16]$} \\
\hline & Lake Kayar & 14.92 & -17.18 & $?$ & [16] \\
\hline & Lake Retba & 14.83 & -17.33 & $?$ & [16] \\
\hline \multirow[t]{14}{*}{ South Africa } & Brandtvlei Saltworks & -30.37 & 20.22 & $?$ & {$[16]$} \\
\hline & Coega Salt Flats & -33.77 & 25.67 & $?(\mathrm{P})$ & [16] \\
\hline & Driehoekspan & -29.75 & 23.23 & $?$ & [16] \\
\hline & Hayfield Saltpan & -29.25 & 24.22 & $?$ & [16] \\
\hline & Holpan Saltworks & -30.37 & 20.50 & $?$ & {$[16]$} \\
\hline & Jonkerwater Saltworks & -30.08 & 22.60 & $?$ & [16] \\
\hline & Kaalpan Saltworks & -30.00 & 20.05 & $?$ & {$[16]$} \\
\hline & Klein Soutpan & -30.45 & 22.40 & $?$ & [16] \\
\hline & Klipfontein Saltworks & -32.95 & 18.22 & $?$ & [16] \\
\hline & Missionvale Salina & -33.87 & 25.53 & $?$ & [16] \\
\hline & Paternoster Salt Pan & -33.78 & 17.92 & $?$ & {$[16]$} \\
\hline & Rietfontein se Pan & -30.27 & 20.12 & $?$ & [16] \\
\hline & Reynekespan Saltworks & -33.68 & 25.78 & $?$ & [16] \\
\hline & Reynekespan & -29.72 & 24.25 & $?$ & {$[16]$} \\
\hline
\end{tabular}




\begin{tabular}{|c|c|c|c|c|c|}
\hline Country & Locality & $\begin{array}{l}\text { Lati- } \\
\text { tude }\end{array}$ & $\begin{array}{c}\text { Longi- } \\
\text { tude }\end{array}$ & $\begin{array}{l}\text { Species (repro- } \\
\text { ductive mode) }\end{array}$ & $\begin{array}{l}\text { Refe- } \\
\text { rence }\end{array}$ \\
\hline & Saldanha Steel & -33.00 & 18.03 & ? & {$[16]$} \\
\hline & Salt Lake & -29.28 & 24.00 & $?$ & {$[16]$} \\
\hline & Sodium Saltworks & -30.17 & 23.13 & $?$ & {$[16]$} \\
\hline & Soutpan area & -28.70 & 26.05 & $?$ & [16] \\
\hline & Soutpan Saltworks & -28.73 & 26.07 & ? & {$[16]$} \\
\hline & Sundays River Saltworks & -33.63 & 25.72 & $?$ & [16] \\
\hline & Swartkops & -33.87 & 25.60 & A. salina $(\mathrm{B})$ & {$[16]$} \\
\hline & Uniesoutpan & -29.60 & 24.43 & $?$ & [16] \\
\hline & Velddrif Saltworks & -32.72 & 18.20 & A. salina $(\mathrm{B})$ & {$[16]$} \\
\hline & Vermeulenspan & -29.75 & 24.33 & $?$ & [16] \\
\hline & Wintersdam Farm & -28.75 & 26.13 & $?$ & [16] \\
\hline & Witkraal Saltpan & -28.97 & 25.52 & $?$ & {$[16]$} \\
\hline & Witpan Saltworks & -29.88 & 24.05 & $?$ & {$[16]$} \\
\hline & Yzerfontein Saltworks & -33.32 & 18.17 & $?$ & {$[16]$} \\
\hline & Zoutaar Saltworks & -30.32 & 23.08 & $?$ & {$[16]$} \\
\hline \multirow[t]{10}{*}{ Tunisia } & Bekalta & 36.80 & 10.33 & A. salina $(\mathrm{B})$ & {$[16] ;[30]$} \\
\hline & COTUSAL salterns & 35.75 & 10.75 & A. salina (B) & J. Muñoz, unpubl. \\
\hline & Chott Ariana & 36.90 & 10.30 & A. salina $(\mathrm{B})$ & {$[16]$} \\
\hline & Chott El Djerid & 33.70 & 8.43 & $?$ & {$[16]$} \\
\hline & Megrine & 36.78 & 10.23 & A. salina $(\mathrm{B})$ & {$[16]$} \\
\hline & Mines Maghreb Salterns & 33.10 & 11.32 & A. salina $(\mathrm{B})$ & J. Muñoz, unpubl. \\
\hline & Sebket Kowezia & 36.43 & 9.77 & $?$ & {$[16]$} \\
\hline & Sebket Mta Moknine & 35.65 & 10.88 & A. salina $(\mathrm{B})$ & {$[16]$} \\
\hline & Sebket Sidi El Hani & 35.52 & 10.45 & $?$ & {$[16]$} \\
\hline & Sfax & 35.75 & 10.72 & A. salina $(\mathrm{B})$ & [16] \\
\hline
\end{tabular}

ASIA

\begin{tabular}{lllclr}
\hline Country & Locality & $\begin{array}{c}\text { Lati- } \\
\text { tude }\end{array}$ & $\begin{array}{c}\text { Longi- } \\
\text { tude }\end{array}$ & $\begin{array}{l}\text { Species (repro- } \\
\text { ductive mode) }\end{array}$ & $\begin{array}{r}\text { Refe- } \\
\text { rence }\end{array}$ \\
\hline Abu Dhabi & Al Wathba Lake & 24.25 & 54.63 & $?$ & {$[30]$} \\
\hline Corea & Pusan & 35.08 & 129.03 & $?$ & {$[24]$} \\
\hline PR China & Aibi & 44.92 & 83.88 & A. parth. 2n (P) & {$[27]$} \\
& Aibi & 44.92 & 83.88 & A. parth. 4n (P) & {$[27]$} \\
& Aletai & 48.00 & 88.00 & $?$ (B) & {$[27]$} \\
& Balikun & 43.50 & 93.00 & A. parth. 2n $(\mathrm{P})$ & {$[27]$} \\
& Balikun & 43.50 & 93.00 & A. parth. 4n $(\mathrm{P})$ & {$[27]$} \\
& Bange & 31.67 & 89.67 & $?$ & {$[27]$} \\
& Bayannor & 44.00 & 116.00 & A. sinica $(\mathrm{B})$ & {$[27]$} \\
& Beidachi & 38.00 & 107.50 & A. sinica $(\mathrm{B})$ & {$[27]$} \\
& Chagannor & 40.00 & 110.00 & A. sinica (B) & {$[27]$} \\
& Chaka & 37.67 & 99.00 & $?$ (P) & {$[27]$} \\
& Chengkou & 38.17 & 117.67 & A. parth. 2n $(\mathrm{P})$ & {$[27]$} \\
\hline
\end{tabular}




\begin{tabular}{|c|c|c|c|c|c|}
\hline Country & Locality & $\begin{array}{l}\text { Lati- } \\
\text { tude }\end{array}$ & $\begin{array}{c}\text { Longi- } \\
\text { tude }\end{array}$ & $\begin{array}{l}\text { Species (repro- } \\
\text { ductive mode) }\end{array}$ & $\begin{array}{l}\text { Refe- } \\
\text { rence }\end{array}$ \\
\hline & Dabancheng & 43.00 & 88.00 & A. parth. $2 \mathrm{n}(\mathrm{P})$ & {$[27]$} \\
\hline & Dabancheng & 43.00 & 88.00 & A. parth. $3 \mathrm{n}(\mathrm{P})$ & [27] \\
\hline & Dabancheng & 43.00 & 88.00 & A. parth. $4 \mathrm{n}(\mathrm{P})$ & [27] \\
\hline & Dabancheng & 43.00 & 88.00 & A. parth. $5 \mathrm{n}(\mathrm{P})$ & [27] \\
\hline & Dacaidan & 37.80 & 95.33 & $?(\mathrm{P})$ & {$[27]$} \\
\hline & Dagenor & 42.50 & 116.00 & A. $\operatorname{sinica}(\mathrm{B})$ & [27] \\
\hline & Daqinghe & 39.83 & 118.83 & $?(\mathrm{P})$ & [27] \\
\hline & Dingbian & 37.67 & 107.50 & $?$ & [27] \\
\hline & Dongcou & 32.17 & 84.67 & $?$ & [27] \\
\hline & Dongfang & 19.03 & 108.92 & $?(\mathrm{P})$ & [30] \\
\hline & Dongfeng & 36.08 & 120.17 & A. parth. $2 \mathrm{n}(\mathrm{P})$ & [27] \\
\hline & Dongfeng & 36.08 & 120.17 & A. parth. $5 \mathrm{n}(\mathrm{P})$ & {$[27]$} \\
\hline & Dongjiagou & 39.33 & 122.00 & A. parth. $2 \mathrm{n}(\mathrm{P})$ & [27] \\
\hline & Ejinor & 45.33 & 112.50 & A. sinica $(\mathrm{B})$ & [27] \\
\hline & Erendabusen & 44.00 & 111.00 & A. sinica $(\mathrm{B})$ & [27] \\
\hline & Fuzhouwan & 39.50 & 121.50 & $?(\mathrm{P})$ & [27] \\
\hline & Gahai & 37.03 & 97.78 & A. parth. $2 \mathrm{n}(\mathrm{P})$ & [27] \\
\hline & Gaize & 32.33 & 84.17 & A. tibetiana (B) & F. Amat, unpubl. \\
\hline & Gaotai & 39.73 & 99.17 & $?(\mathrm{~B})$ & {$[32]$} \\
\hline & Geji & 32.40 & 81.17 & $?$ & [27] \\
\hline & Guangdong & 22.87 & 113.47 & $?(\mathrm{P})$ & [27] \\
\hline & Hangjinqi & 40.00 & 101.00 & A. sinica $(\mathrm{B})$ & [27] \\
\hline & Hangu & 39.42 & 117.83 & A. parth. $2 \mathrm{n}(\mathrm{P})$ & [27] \\
\hline & Haolebaoji & 38.90 & 108.50 & A. sinica $(\mathrm{B})$ & [27] \\
\hline & Haotongyin Chagan & 39.17 & 108.92 & A. sinica $(\mathrm{B})$ & {$[27]$} \\
\hline & Huanghua & 38.33 & 117.67 & A. parth. $2 \mathrm{n}(\mathrm{P})$ & {$[27]$} \\
\hline & Huanghua & 38.33 & 117.67 & A. franciscana (B) & [31] \\
\hline & Huhetaolergai & 37.00 & 110.00 & A. sinica $(\mathrm{B})$ & {$[27]$} \\
\hline & Jibuchaka & 32.00 & 84.17 & $?$ & [27] \\
\hline & Jilantai & 39.80 & 103.60 & A. sinica $(\mathrm{B})$ & [27] \\
\hline & Jimo & 36.38 & 120.45 & $?(\mathrm{P})$ & {$[30]$} \\
\hline & Jinzhou & 40.80 & 121.00 & $?(\mathrm{P})$ & [27] \\
\hline & Kangbao & 41.80 & 114.60 & A. sinica $(\mathrm{B})$ & {$[27]$} \\
\hline & Keke & 37.00 & 98.00 & A. parth. $4 \mathrm{n}(\mathrm{P})$ & {$[27]$} \\
\hline & Lagkor Co & 32.05 & 84.22 & A. tibetiana (B) & {$[2]$} \\
\hline & Lianyungang & 34.67 & 119.50 & $?(\mathrm{P})$ & {$[27]$} \\
\hline & Luannan & 39.17 & 118.50 & $?(\mathrm{P})$ & {$[31]$} \\
\hline & Luannan & 39.17 & 118.50 & A. franciscana (B) & {$[31]$} \\
\hline & Luannan & 39.17 & 118.50 & A. sinica $(\mathrm{B})$ & {$[31]$} \\
\hline & Lushun & 30.83 & 121.33 & A. parth. $2 \mathrm{n}(\mathrm{P})$ & [27] \\
\hline & Lushun & 30.83 & 121.33 & A. parth. $4 \mathrm{n}(\mathrm{P})$ & [27] \\
\hline & Lushun & 30.83 & 121.33 & A. parth. 5n (P) & [27] \\
\hline & Nanpu & 39.08 & 118.33 & A. parth. $2 \mathrm{n}(\mathrm{P})$ & {$[27]$} \\
\hline & Nanwan & 32.15 & 113.98 & $?(\mathrm{P})$ & {$[30]$} \\
\hline & Pulandian & 39.00 & 122.00 & A. parth. $2 \mathrm{n}(\mathrm{P})$ & [27] \\
\hline
\end{tabular}




\begin{tabular}{|c|c|c|c|c|c|}
\hline Country & Locality & $\begin{array}{l}\text { Lati- } \\
\text { tude }\end{array}$ & $\begin{array}{l}\text { Longi- } \\
\text { tude }\end{array}$ & $\begin{array}{l}\text { Species (repro- } \\
\text { ductive mode) }\end{array}$ & $\begin{array}{l}\text { Refe- } \\
\text { rence }\end{array}$ \\
\hline & Sanggendalai & 42.33 & 116.00 & A. sinica $(\mathrm{B})$ & {$[27]$} \\
\hline & Shangyi & 41.10 & 114.68 & A. sinica $(\mathrm{B})$ & {$[27]$} \\
\hline & Shanyao & 25.13 & 118.88 & $?(\mathrm{P})$ & {$[27]$} \\
\hline & Shenzha & 31.00 & 88.67 & $?(\mathrm{~B})$ & [27] \\
\hline & Shunmu & 29.83 & 122.25 & $?(\mathrm{P})$ & {$[27]$} \\
\hline & Suban & 39.00 & 94.00 & $?(\mathrm{P})$ & [27] \\
\hline & Taigemiao Chagan & 39.08 & 109.92 & A. sinica $(\mathrm{B})$ & [27] \\
\hline & Tanggu & 39.00 & 117.67 & A. franciscana $(\mathrm{B})$ & {$[31]$} \\
\hline & Tanggu & 39.00 & 117.67 & A. sinica $(\mathrm{B})$ & [31] \\
\hline & Tanggu & 39.00 & 117.67 & $?(\mathrm{P})$ & {$[31]$} \\
\hline & Tuosu & 37.17 & 96.90 & $?(\mathrm{P})$ & [27] \\
\hline & Wumacou & 32.50 & 83.17 & $?$ & {$[27]$} \\
\hline & Wuqiangi & 41.00 & 109.00 & A. sinica $(\mathrm{B})$ & {$[27]$} \\
\hline & Xiaocaidan & 37.00 & 95.10 & $?(\mathrm{P})$ & [27] \\
\hline & Xiaotan & 22.67 & 113.22 & $?(\mathrm{P})$ & {$[30]$} \\
\hline & Xigang & 23.42 & 117.92 & $?(\mathrm{P})$ & [27] \\
\hline & Xuyu & 34.60 & 113.60 & $?(\mathrm{P})$ & [27] \\
\hline & Yangkou & 37.33 & 119.00 & A. parth. $2 \mathrm{n}(\mathrm{P})$ & [27] \\
\hline & Yanjing & 29.00 & 98.50 & $?(\mathrm{~B})$ & [27] \\
\hline & Yinggehai & 18.50 & 108.60 & A. parth. $2 \mathrm{n}(\mathrm{P})$ & [27] \\
\hline & Yinggehai & 18.50 & 108.60 & A. parth. $4 \mathrm{n}(\mathrm{P})$ & [27] \\
\hline & Yinggehai & 18.50 & 108.60 & A. parth. 5n (P) & {$[27]$} \\
\hline & Yingkou & 40.67 & 122.00 & A. parth. $2 \mathrm{n}(\mathrm{P})$ & [27] \\
\hline & Yingkou & 40.67 & 122.00 & A. parth. 4n (P) & [27] \\
\hline & Yingkou & 40.67 & 122.00 & A. parth. $5 \mathrm{n}(\mathrm{P})$ & {$[27]$} \\
\hline & Yuncheng & 35.00 & 111.00 & A. sinica $(\mathrm{B})$ & F. Amat, unpubl. \\
\hline & Zhangbei & 41.17 & 114.70 & A. sinica $(\mathrm{B})$ & {$[27]$} \\
\hline & Zhangchaka & 32.78 & 82.38 & $?$ & [27] \\
\hline & Zhanmao & 30.52 & 122.30 & $?(\mathrm{P})$ & {$[27]$} \\
\hline & Zhujiajian & 29.83 & 122.38 & $?(\mathrm{P})$ & [27] \\
\hline & Zhunsaihannor & 43.00 & 115.00 & A. sinica $(\mathrm{B})$ & [27] \\
\hline \multirow[t]{15}{*}{ India } & Balamba Salterns & 23.40 & 70.28 & $?(\mathrm{P})$ & [27] \\
\hline & Bhayander & 18.92 & 72.83 & $?(\mathrm{P})$ & {$[24]$} \\
\hline & Didwana & 27.05 & 74.08 & $?$ & [27] \\
\hline & Gulf of Kutch & 23.33 & 71.00 & $?(\mathrm{P})$ & [27] \\
\hline & Jamnagar & 22.50 & 70.13 & $?$ & [27] \\
\hline & Karsewar Island & 8.83 & 78.17 & $?$ & {$[27]$} \\
\hline & Kelambakkam & 13.08 & 79.12 & $?$ & [27] \\
\hline & Mithapur & 23.00 & 70.17 & $?(\mathrm{P})$ & {$[27]$} \\
\hline & Pattanamaruther & 8.92 & 78.13 & $?$ & {$[27]$} \\
\hline & Sambhar Lake & 26.90 & 75.17 & $?$ & {$[30]$} \\
\hline & Spic Nagar and Thiespuram & 8.83 & 78.13 & A. franciscana $(\mathrm{B})$ & F. Amat, unpubl. \\
\hline & Thamaraikulam & 8.08 & 77.52 & $?(\mathrm{P})$ & {$[27]$} \\
\hline & Vadala and Bahinder & 18.92 & 72.83 & $?$ & {$[27]$} \\
\hline & Vedaranyam & 10.02 & 79.83 & $?$ & {$[27]$} \\
\hline & Veppalodai & 8.98 & 78.13 & A. franciscana $(\mathrm{B})$ & F. Amat, unpubl. \\
\hline
\end{tabular}




\begin{tabular}{|c|c|c|c|c|c|}
\hline Country & Locality & $\begin{array}{l}\text { Lati- } \\
\text { tude }\end{array}$ & $\begin{array}{l}\text { Longi- } \\
\text { tude }\end{array}$ & $\begin{array}{l}\text { Species (repro- } \\
\text { ductive mode) }\end{array}$ & $\begin{array}{l}\text { Refe- } \\
\text { rence }\end{array}$ \\
\hline \multirow[t]{4}{*}{ Iraq } & Abu-Graib, & 33.33 & 44.50 & A. parth. 2n (P) & F. Amat, unpubl. \\
\hline & Basra & 30.42 & 47.85 & A. franciscana $(\mathrm{B})$ & F. Amat, unpubl. \\
\hline & Dayala & 33.50 & 44.50 & $?$ & {$[27]$} \\
\hline & Mahmoodia & 33.00 & 44.00 & $?$ & {$[27]$} \\
\hline \multirow[t]{21}{*}{ Iran } & Athlit & 32.70 & 34.93 & $?$ & {$[27]$} \\
\hline & Bakhtegan Lake & 29.67 & 53.83 & $?(\mathrm{P})$ & {$[1]^{*}$} \\
\hline & Gaav Khooni Lake & 32.33 & 52.97 & $?(\mathrm{P})$ & {$[1]^{*}$} \\
\hline & Houze Sultan Lake & 34.83 & 51.33 & $?(\mathrm{P})$ & {$[1]^{*}$} \\
\hline & Incheh Lake & 37.40 & 54.60 & $?(\mathrm{P})$ & {$[1]^{*}$} \\
\hline & Kale Shoor Hashtgerd & 36.50 & 51.30 & $?(\mathrm{P})$ & {$[1]^{*}$} \\
\hline & Kale Shoor, Gonabad & 35.17 & 57.83 & $?(\mathrm{P})$ & {$[1]^{*}$} \\
\hline & Kale Shoor, Khorram Abad & 32.67 & 48.90 & $?(\mathrm{P})$ & {$[1]^{*}$} \\
\hline & Lagoons around Urmia Lake & 37.83 & 46.67 & $?(\mathrm{P})$ & {$[1]^{*}$} \\
\hline & Maharlu Lake & 29.95 & 52.23 & $?(\mathrm{P})$ & {$[1]^{*}$} \\
\hline & Mighan Lake & 34.33 & 50.33 & $?(\mathrm{P})$ & {$[1]^{*}$} \\
\hline & Nough Catchment & 31.00 & 56.83 & $?(\mathrm{P})$ & {$[1]^{*}$} \\
\hline & Nough Catchment & 31.00 & 56.83 & A. franciscana $(\mathrm{B})$ & {$[1]^{*}$} \\
\hline & Qom Salt Lake & 34.67 & 52.33 & $?(\mathrm{P})$ & {$[1]^{*}$} \\
\hline & Schor-Gol & 37.05 & 45.53 & $?(\mathrm{P})$ & [27] \\
\hline & Shor Lake & 37.42 & 54.68 & $?(\mathrm{P})$ & {$[1]^{*}$} \\
\hline & Shurabil Lake & 38.42 & 48.92 & $?(\mathrm{P})$ & {$[1]^{*}$} \\
\hline & Tashk Lake & 30.00 & 53.83 & $?(\mathrm{P})$ & {$[1]^{*}$} \\
\hline & Urmia Lake & 37.33 & 45.67 & A. urmiana (B) & [27] \\
\hline & Urmia Lake & 37.33 & 45.67 & $?(\mathrm{P})$ & {$[1]^{*}$} \\
\hline & Varmal Catchment & 31.33 & 61.83 & $?(\mathrm{P})$ & {$[1]^{*}$} \\
\hline \multirow[t]{2}{*}{ Israel } & Eilat North & 29.53 & 34.93 & $?(\mathrm{P})$ & [27] \\
\hline & Eilat South & 29.47 & 34.93 & $?$ & [27] \\
\hline \multirow[t]{3}{*}{ Japan } & Chang Dao & 34.00 & 132.00 & $?$ & {$[27]$} \\
\hline & Tamano & 34.88 & 133.98 & $?$ & [27] \\
\hline & Yamaguchi & 34.17 & 131.53 & $?$ & [27] \\
\hline \multirow[t]{14}{*}{ Kazakhstan } & Bjurliu lake & 51.75 & 78.00 & A. parth. $2 \mathrm{n}(\mathrm{P})$ & J. Muñoz, unpubl. \\
\hline & $\begin{array}{l}\text { Bolshoy Sarichaganack, } \\
\text { Aral Sea }\end{array}$ & 46.50 & 61.25 & $?$ & {$[20]$} \\
\hline & Borli & 51.82 & 78.00 & $?(\mathrm{P})$ & [20] \\
\hline & Horbatoye & 45.50 & 73.50 & $?(\mathrm{P})$ & {$[20]$} \\
\hline & Kalibek & 53.87 & 70.63 & $?$ & {$[20]$} \\
\hline & Miraldi & 52.32 & 77.78 & $?(\mathrm{P})$ & [20] \\
\hline & Severo-zapadnoye & 45.50 & 73.33 & $?(\mathrm{P})$ & [20] \\
\hline & Seyten & 51.93 & 78.12 & $?(\mathrm{P})$ & {$[20]$} \\
\hline & Shoshkakol & 49.17 & 70.50 & $?$ & [20] \\
\hline & Tastubeck, Aral Sea & 46.83 & 60.75 & $?$ & [20] \\
\hline & Teke & 53.83 & 72.93 & $?$ & {$[20]$} \\
\hline & Tenhiz & 50.42 & 69.00 & $?$ & [20] \\
\hline & Tuz & 51.32 & 78.65 & $?$ & [20] \\
\hline & Yuzhnoye & 44.92 & 74.17 & $?(\mathrm{P})$ & [20] \\
\hline
\end{tabular}




\begin{tabular}{|c|c|c|c|c|c|}
\hline Country & Locality & $\begin{array}{l}\text { Lati- } \\
\text { tude }\end{array}$ & $\begin{array}{l}\text { Longi- } \\
\text { tude }\end{array}$ & $\begin{array}{l}\text { Species (repro- } \\
\text { ductive mode) }\end{array}$ & $\begin{array}{l}\text { Refe- } \\
\text { rence }\end{array}$ \\
\hline Kuwait & $?$ & 29.00 & 47.00 & $?$ & {$[27]$} \\
\hline Mongolia & Shar Burd & 43.37 & 111.07 & $?$ & {$[30]$} \\
\hline Pakistan & Karachi Saltworks & 24.80 & 66.97 & $?(\mathrm{P})$ & [27] \\
\hline Saudi Arabia & $\begin{array}{l}\text { Dhahran } \\
\text { Sabkhat al Fasl }\end{array}$ & $\begin{array}{l}17.65 \\
27.03\end{array}$ & $\begin{array}{l}43.50 \\
49.50\end{array}$ & $\begin{array}{l}? \\
?\end{array}$ & $\begin{array}{l}{[30]} \\
{[30]}\end{array}$ \\
\hline South Korea & Pusan & 35.08 & 129.03 & $?$ & [27] \\
\hline Sri Lanka & $\begin{array}{l}\text { Bundala } \\
\text { Hambantota } \\
\text { Palavi } \\
\text { Putallam }\end{array}$ & $\begin{array}{l}6.20 \\
6.12 \\
7.97 \\
8.03 \\
\end{array}$ & $\begin{array}{l}81.25 \\
81.12 \\
79.85 \\
79.83 \\
\end{array}$ & $\begin{array}{l}? \\
? \\
? \\
?(\mathrm{P})\end{array}$ & $\begin{array}{l}{[27]} \\
{[27]} \\
{[27]} \\
{[27]} \\
\end{array}$ \\
\hline Taiwan & Beimen & 23.33 & 121.12 & $?(\mathrm{~B})$ & {$[27]$} \\
\hline Turkey & $\begin{array}{l}\text { Ayvalik, Balikesir } \\
\text { Burdur Lake } \\
\text { Camalti, Izmir } \\
\text { Gökçeada, Imroz } \\
\text { Konya Lake } \\
\text { Meke Salt lake } \\
\text { Tuz Lake }\end{array}$ & $\begin{array}{l}39.30 \\
37.75 \\
38.42 \\
40.17 \\
37.87 \\
37.68 \\
38.75\end{array}$ & $\begin{array}{l}26.70 \\
30.25 \\
27.13 \\
25.83 \\
32.47 \\
33.63 \\
33.37\end{array}$ & $\begin{array}{l}? \\
? \\
?(\mathrm{P}) \\
? \\
? \\
? \\
?(\mathrm{P})\end{array}$ & $\begin{array}{l}{[30]} \\
{[30]} \\
{[27]} \\
{[30]} \\
{[30]} \\
{[30]} \\
{[10]}\end{array}$ \\
\hline Turkmenistan & Karabogaz Lake & 40.98 & 52.95 & $?(\mathrm{P})$ & [30] \\
\hline Uzbekistan & $\begin{array}{l}\text { Adzibay Bight, Aral Sea } \\
\text { Cape Aktumsyk, Aral Sea } \\
\text { Karshi, Hashkadarya } \\
\text { Navruz, Syrdarya } \\
\text { Nazurok } \\
\text { Ulugshurkul }\end{array}$ & $\begin{array}{l}43.97 \\
44.60 \\
38.82 \\
40.82 \\
41.50 \\
41.33\end{array}$ & $\begin{array}{l}58.58 \\
58.30 \\
65.82 \\
68.67 \\
60.17 \\
60.50\end{array}$ & $\begin{array}{l}\text { ? } \\
\text { A. parth. 2n }(\mathrm{P}) \\
?(\mathrm{P}) \\
?(\mathrm{P}) \\
? \\
?\end{array}$ & $\begin{array}{l}{[20]} \\
{[19]} \\
{[30]} \\
{[30]} \\
{[20]} \\
{[20]}\end{array}$ \\
\hline
\end{tabular}

\section{AUSTRALIA AND NEW ZEALAND}

\begin{tabular}{llcclr}
\hline Country & Locality & $\begin{array}{c}\text { Lati- } \\
\text { tude }\end{array}$ & $\begin{array}{c}\text { Longi- } \\
\text { tude }\end{array}$ & $\begin{array}{l}\text { Species (repro- } \\
\text { ductive mode) }\end{array}$ & $\begin{array}{r}\text { Refe- } \\
\text { rence }\end{array}$ \\
\hline New Zealand & Lake Grassmere & -41.63 & 174.08 & A. franciscana $(\mathrm{B})$ & {$[27]$} \\
\hline Australia & Bowen & -20.00 & 148.27 & $?$ & {$[27]$} \\
& Dampier & -20.58 & 116.85 & A. franciscana $(\mathrm{B})$ & {$[27]$} \\
& Dry Creek, Adelaide & -34.92 & 138.33 & ? (P) & {$[27]$} \\
& Goodmalling & -31.08 & 117.03 & A. parth. 2n $(\mathrm{P})$ & {$[18]$} \\
& Hutt River & -28.20 & 114.30 & A. franciscana $(\mathrm{B})$ & {$[18]$} \\
& Lake Hayward & -28.43 & 114.77 & A. parth. 2n $(\mathrm{P})$ & {$[18]$} \\
& Lake Mc Leod & -23.98 & 113.67 & $?$ & {$[27]$} \\
& Lake Ninan & -30.93 & 116.63 & A. parth. 2n $(\mathrm{P})$ & {$[18]$} \\
\hline
\end{tabular}




\begin{tabular}{llcclr}
\hline Country & Locality & $\begin{array}{c}\text { Lati- } \\
\text { tude }\end{array}$ & $\begin{array}{c}\text { Longi- } \\
\text { tude }\end{array}$ & $\begin{array}{l}\text { Species (repro- } \\
\text { ductive mode) }\end{array}$ & $\begin{array}{r}\text { Refe- } \\
\text { rence }\end{array}$ \\
\hline & Port Alma & -23.37 & 150.53 & A. franciscana $(\mathrm{B})$ & {$[27]$} \\
& Port Hedland & -20.42 & 118.58 & $?(\mathrm{P})$ & {$[27]$} \\
Quairading & -32.00 & 117.40 & A. parth. 2n $(\mathrm{P})$ & {$[18]$} \\
Rottnest Island & -32.00 & 115.45 & $?(\mathrm{P})$ & {$[27]$} \\
& Shark Bay & -25.25 & 113.33 & A. franciscana $(\mathrm{B})$ & {$[27]$} \\
& Shark Bay & -25.25 & 113.33 & $?(\mathrm{P})$ & {$[27]$} \\
\hline
\end{tabular}

\section{CARIBBEAN_CENTRAL_SOUTH AMERICA}

\begin{tabular}{|c|c|c|c|c|c|}
\hline Country & Locality & $\begin{array}{l}\text { Lati- } \\
\text { tude }\end{array}$ & $\begin{array}{l}\text { Longi- } \\
\text { tude }\end{array}$ & $\begin{array}{l}\text { Species (repro- } \\
\text { ductive mode) }\end{array}$ & $\begin{array}{l}\text { Refe- } \\
\text { rence }\end{array}$ \\
\hline \multicolumn{6}{|c|}{ Caribbean and Central America } \\
\hline \multirow{3}{*}{ Bahamas } & Great Inagua & 21.00 & -75.33 & ?(B) & [27] \\
\hline & Long Island & 23.33 & -75.12 & $?(\mathrm{~B})$ & [27] \\
\hline & San Salvador & 24.00 & -74.58 & $?(\mathrm{~B})$ & [27] \\
\hline Brit. Virgin Islands & Anegada & 18.75 & -64.40 & $?(\mathrm{~B})$ & [27] \\
\hline \multirow[t]{4}{*}{ Caribbian Islands } & Antigua & 17.00 & -61.75 & $?(\mathrm{~B})$ & [27] \\
\hline & South Caicos & 21.52 & -71.53 & $?(\mathrm{~B})$ & [27] \\
\hline & St. Kitts & 17.33 & -62.75 & $?(\mathrm{~B})$ & [27] \\
\hline & St. Martin & 18.07 & -63.10 & $?(\mathrm{~B})$ & [27] \\
\hline \multirow[t]{2}{*}{ Costa Rica } & Gulfo Nicova & 10.00 & -84.82 & $?(\mathrm{~B})$ & [27] \\
\hline & Bahia Salinas, Guanacaste & 10.02 & -85.67 & A. franciscana (B) & [22] \\
\hline Dominican & Isla Cabra & 19.88 & -71.67 & ?(B) & {$[27]$} \\
\hline \multirow[t]{3}{*}{ Republic } & Las Calderas & 18.20 & -70.55 & $?(\mathrm{~B})$ & [30] \\
\hline & Monte Cristi & 19.87 & -71.65 & $?(\mathrm{~B})$ & [27] \\
\hline & Punta Salinas & 18.33 & -71.07 & $?(\mathrm{~B})$ & [27] \\
\hline Haiti & Grandes Salines & 18.00 & -72.00 & A. franciscana (B) & [27] \\
\hline \multirow[t]{16}{*}{ Mexico } & Bahia de Ceuta & 24.08 & -107.00 & A. franciscana $(\mathrm{B})$ & {$[27]$} \\
\hline & Carretas/Pereyra/Chanchuto & 15.50 & -93.22 & $?(\mathrm{~B})$ & [27] \\
\hline & Celestun & 20.87 & -90.37 & $?(\mathrm{~B})$ & {$[25]$} \\
\hline & Chuburna & 21.25 & -89.80 & $?(\mathrm{~B})$ & [25] \\
\hline & Guerrero Negro & 28.10 & -114.05 & A. franciscana $(\mathrm{B})$ & [27] \\
\hline & Isla del Carmen & 26.00 & -111.67 & A. franciscana $(\mathrm{B})$ & [27] \\
\hline & La Joya y Buenavista & 27.45 & -106.25 & $?(\mathrm{~B})$ & [27] \\
\hline & Laguna de Yavaros & 26.72 & -109.55 & A. franciscana $(\mathrm{B})$ & [27] \\
\hline & Laguna del Mar Muerto & 16.00 & -94.00 & $?(\mathrm{~B})$ & [27] \\
\hline & Las Coloradas & 21.60 & -87.98 & $?(\mathrm{~B})$ & [25] \\
\hline & Pichilingue & 24.28 & -110.33 & A. franciscana $(\mathrm{B})$ & [27] \\
\hline & Ponds W. Salina Cruz & 16.17 & -95.17 & $?(\mathrm{~B})$ & [27] \\
\hline & Salinas de Hidalgo & 22.67 & -101.70 & $?(\mathrm{~B})$ & [27] \\
\hline & San Crisanto & 24.08 & -107.00 & A. franciscana $(\mathrm{B})$ & {$[27]$} \\
\hline & San Quintin & 30.47 & -115.97 & A. franciscana $(\mathrm{B})$ & [27] \\
\hline & Xtampu & 21.38 & -88.88 & $?(\mathrm{~B})$ & [25] \\
\hline
\end{tabular}




\begin{tabular}{|c|c|c|c|c|c|}
\hline Country & Locality & $\begin{array}{l}\text { Lati- } \\
\text { tude }\end{array}$ & $\begin{array}{c}\text { Longi- } \\
\text { tude }\end{array}$ & $\begin{array}{l}\text { Species (repro- } \\
\text { ductive mode) }\end{array}$ & $\begin{array}{l}\text { Refe- } \\
\text { rence }\end{array}$ \\
\hline \multirow{8}{*}{$\begin{array}{c}\text { Netherlands } \\
\text { Antilles }\end{array}$} & Aruba & 12.50 & -70.00 & $?(\mathrm{~B})$ & {$[27]$} \\
\hline & Bonaire Duinmeer & 12.07 & -68.22 & A. franciscana (B) & [27] \\
\hline & Curaçao Fuik & 12.05 & -68.85 & $?(\mathrm{~B})$ & [27] \\
\hline & Gotomeer & 12.23 & -68.33 & $?(\mathrm{~B})$ & [27] \\
\hline & Martinus & 12.15 & -68.28 & $?(\mathrm{~B})$ & [27] \\
\hline & Pekelmeer & 12.07 & -68.27 & $?(\mathrm{~B})$ & {$[27]$} \\
\hline & Rifwater & 12.13 & -68.33 & $?(\mathrm{~B})$ & [27] \\
\hline & Slagbaai & 12.27 & -68.42 & $?(\mathrm{~B})$ & [27] \\
\hline Nicaragua & Salinas Grandes, Leon & 12.25 & -85.85 & A. franciscana (B) & {$[30]$} \\
\hline \multirow{6}{*}{ Puerto Rico } & Bahia Salinas & 17.95 & -67.20 & A. franciscana $(\mathrm{B})$ & {$[27]$} \\
\hline & Bogueron & 18.02 & -67.17 & $?(\mathrm{~B})$ & [27] \\
\hline & Cabo Rojo and Fortuna saltern & 17.93 & -67.13 & $?(\mathrm{~B})$ & {$[17] ;[27$} \\
\hline & La Parguera and Bastoncillo & 17.98 & -67.05 & $?(\mathrm{~B})$ & {$[17] ;[27$} \\
\hline & Ponce & 19.10 & -66.63 & $?(\mathrm{~B})$ & [27] \\
\hline & Tallaboa Salterns & 17.97 & -66.70 & A. franciscana (B) & {$[27]$} \\
\hline \multicolumn{6}{|c|}{ South America } \\
\hline \multirow[t]{21}{*}{ Argentina } & Algarrobo & -40.60 & -62.93 & A. persimilis (B) & [23] \\
\hline & Bahia Blanca & -38.72 & -62.25 & A. persimilis (B) & [27] \\
\hline & Caleta Olivia & -46.45 & -67.53 & A. persimilis (B) & [23] \\
\hline & El Ingles & -40.72 & -62.47 & A. persimilis (B) & [23] \\
\hline & El Saladillo & -28.33 & -63.17 & A. franciscana $(\mathrm{B})$ & [23] \\
\hline & Gualicho & -40.40 & -65.22 & A. persimilis (B) & {$[23]$} \\
\hline & Laguna Callaqueo & -38.57 & -63.53 & A. persimilis (B) & {$[13] ;[23$} \\
\hline & Las Tunas & -33.75 & -62.53 & A. franciscana $(\mathrm{B})$ & [23] \\
\hline & Mar Chiquita & -30.33 & -62.17 & A. franciscana (B) & [23] \\
\hline & Palos Blancos & -39.47 & -62.75 & A. persimilis $(\mathrm{B})$ & [23] \\
\hline & Piedras & -40.68 & -62.67 & A. persimilis (B) & {$[23]$} \\
\hline & Ruta 3 & -47.47 & -67.27 & A. persimilis (B) & {$[23]$} \\
\hline & Salina Colorada Chica & -38.38 & -63.60 & A. persimilis (B) & [23] \\
\hline & Salina Colorada Grande & -38.30 & -63.70 & A. persimilis (B) & [23] \\
\hline & Salinas Chicas & -38.72 & -62.93 & A. persimilis (B) & [23] \\
\hline & Salinas Grandes de Anzoatequi & -38.98 & -63.87 & ? & {$[30]$} \\
\hline & Salinas Grandes de Hidalgo & -37.22 & -63.43 & A. persimilis $(\mathrm{B})$ & {$[23]$} \\
\hline & Salitral de la Vidriera & -38.70 & -62.67 & A. persimilis (B) & [23] \\
\hline & Salitral Negro & -38.73 & -63.22 & A. persimilis (B) & {$[23]$} \\
\hline & San Julian & -49.28 & -67.77 & A. persimilis (B) & {$[23]$} \\
\hline & Valcheta, Rio Negro & -40.68 & -66.12 & ? & {$[30]$} \\
\hline \multirow[t]{4}{*}{ Bolivia } & Chulluncani & -16.37 & -67.50 & $?(\mathrm{~B})$ & {$[24] ;[29$} \\
\hline & Lake Kanapa & -16.87 & -68.87 & $?(\mathrm{~B})$ & [30] \\
\hline & Lake Poopo & -18.38 & -66.97 & $?(\mathrm{~B})$ & [27] \\
\hline & Papel Pampa, Oruro & -17.82 & -67.77 & $?(\mathrm{~B})$ & [30] \\
\hline
\end{tabular}




\begin{tabular}{|c|c|c|c|c|c|}
\hline Country & Locality & $\begin{array}{l}\text { Lati- } \\
\text { tude }\end{array}$ & $\begin{array}{l}\text { Longi- } \\
\text { tude }\end{array}$ & $\begin{array}{l}\text { Species (repro- } \\
\text { ductive mode) }\end{array}$ & $\begin{array}{l}\text { Refe- } \\
\text { rence }\end{array}$ \\
\hline \multirow[t]{12}{*}{ Brazil } & Aracati, Ceará & -4.53 & -37.75 & A. franciscana (B) & [27] \\
\hline & Areia Branca & -10.75 & -37.07 & A. franciscana (B) & {$[11]^{*}$} \\
\hline & Cabo Frio & -22.85 & -42.05 & A. franciscana (B) & {$[27]$} \\
\hline & Camocim, Ceará & -2.90 & -40.83 & A. franciscana (B) & {$[30]$} \\
\hline & Fortaleza & -3.75 & -38.58 & $?$ & {$[24]$} \\
\hline & Galinhos & -5.10 & -36.27 & A. franciscana $(\mathrm{B})$ & [11] \\
\hline & Guamaré & -5.08 & -36.32 & A. franciscana (B) & [11] \\
\hline & Grossos & -4.97 & -37.15 & A. franciscana (B) & [11] \\
\hline & Icapuí, Ceará & -4.70 & -37.35 & A. franciscana (B) & {$[27]$} \\
\hline & Macau & -5.00 & -36.67 & A. franciscana (B) & [11] \\
\hline & Mundau & -3.25 & -39.40 & A. franciscana (B) & {$[24] ;[27]$} \\
\hline & Sao Bento do Norte & -5.10 & -35.97 & A. franciscana (B) & [30] \\
\hline \multirow[t]{16}{*}{ Chile } & Cejas Lagoon & -23.03 & -68.22 & A. franciscana (B) & {$[33]$} \\
\hline & La Pampilla & -29.97 & -71.42 & A. franciscana (B) & {$[27]$} \\
\hline & Laguna Amarga & -50.97 & -72.75 & A. persimilis (B) & {$[12]$} \\
\hline & Los Vilos & -31.85 & -71.42 & A. franciscana (B) & {$[14]$} \\
\hline & Palo Colorado & -31.97 & -71.42 & A. franciscana (B) & [27] \\
\hline & Pampilla & -29.83 & -71.37 & A. franciscana (B) & [14] \\
\hline & Pichilemu & -34.80 & -72.17 & A. franciscana (B) & {$[33]$} \\
\hline & Playa Yape & -20.67 & -70.25 & A. franciscana (B) & {$[27]$} \\
\hline & Puerto Viejo & -27.33 & -70.95 & A. franciscana $(\mathrm{B})$ & {$[27]$} \\
\hline & Salar de Atacama & -23.17 & -68.17 & A. franciscana (B) & [27] \\
\hline & Salar de Llamara & -21.30 & -69.62 & A. franciscana $(\mathrm{B})$ & {$[27]$} \\
\hline & Salar de Pintados & -21.50 & -69.67 & A. franciscana (B) & {$[27]$} \\
\hline & Salar de Surire & -18.80 & -69.07 & A. franciscana $(\mathrm{B})$ & {$[33]$} \\
\hline & Salina El Convento & -33.87 & -71.73 & $?(\mathrm{~B})$ & [14] \\
\hline & Salinas de Cahuil & -34.42 & -72.17 & A. franciscana $(\mathrm{B})$ & {$[27]$} \\
\hline & Salinas de Constitución & -35.15 & -72.35 & A. franciscana (B) & {$[27]$} \\
\hline \multirow[t]{2}{*}{ Colombia } & Galerazamba & -10.42 & -74.67 & A. franciscana (B) & [27] \\
\hline & Manaure & -12.15 & -71.92 & A. franciscana (B) & {$[27]$} \\
\hline \multirow[t]{3}{*}{ Ecuador } & Galapagos & 0.00 & -89.00 & A. franciscana $(\mathrm{B})$ & {$[27]$} \\
\hline & Pacoa & -2.00 & -80.83 & $?(\mathrm{~B})$ & [27] \\
\hline & Salinas & -2.33 & -80.97 & $?(\mathrm{~B})$ & [27] \\
\hline \multirow[t]{9}{*}{ Peru } & Ancash & -9.38 & -77.70 & A. franciscana (B) & {$[30]$} \\
\hline & Caucato & -13.67 & -76.08 & $?(\mathrm{~B})$ & {$[27]$} \\
\hline & Chicama & -7.70 & -80.20 & $?(\mathrm{~B})$ & {$[27]$} \\
\hline & Chilca & -12.58 & -76.68 & $?(\mathrm{~B})$ & [27] \\
\hline & Estuario de Virrila & -5.83 & -80.83 & $?(\mathrm{~B})$ & {$[27]$} \\
\hline & Guadalupe & -7.28 & -79.47 & $?(\mathrm{~B})$ & [27] \\
\hline & Pampa de Salinas y Playa Chica & -11.23 & -77.58 & $?(\mathrm{~B})$ & [27] \\
\hline & Puerto Huarmey & -10.05 & -78.13 & $?(\mathrm{~B})$ & {$[27]$} \\
\hline & Tumbes & -3.62 & -80.45 & $?(\mathrm{~B})$ & {$[27]$} \\
\hline
\end{tabular}




\begin{tabular}{lllllr}
\hline Country & Locality & $\begin{array}{c}\text { Lati- } \\
\text { tude }\end{array}$ & $\begin{array}{c}\text { Longi- } \\
\text { tude }\end{array}$ & $\begin{array}{l}\text { Species (repro- } \\
\text { ductive mode) }\end{array}$ & $\begin{array}{r}\text { Refe- } \\
\text { rence }\end{array}$ \\
\hline Venezuela & Boca Chica & 10.95 & -64.43 & $?(\mathrm{~B})$ & {$[27]$} \\
& Coya Sal & 10.93 & -68.25 & $?(\mathrm{~B})$ & {$[27]$} \\
& Coche & 10.68 & -63.97 & $?(\mathrm{~B})$ & {$[27]$} \\
& Coro Coastline & 11.50 & -69.75 & $?(\mathrm{~B})$ & {$[27]$} \\
& Cumaná & 10.45 & -64.17 & $?(\mathrm{~B})$ & {$[30]$} \\
& La Orchila & 11.82 & -66.00 & $?(\mathrm{~B})$ & {$[27]$} \\
& Las Aves & 12.00 & -67.28 & $?(\mathrm{~B})$ & {$[27]$} \\
& Los Roques & 11.83 & -66.63 & $?(\mathrm{~B})$ & {$[30]$} \\
& Maracaibo Lake & 10.63 & -71.63 & $?(\mathrm{~B})$ & {$[27]$} \\
& Port Araya & 10.65 & -64.28 & $?(\mathrm{~B})$ & {$[27]$} \\
\hline
\end{tabular}

\section{EUROPE}

\begin{tabular}{|c|c|c|c|c|c|}
\hline Country & Locality & $\begin{array}{l}\text { Lati- } \\
\text { tude }\end{array}$ & $\begin{array}{l}\text { Longi- } \\
\text { tude }\end{array}$ & $\begin{array}{l}\text { Species (repro- } \\
\text { ductive mode) }\end{array}$ & $\begin{array}{l}\text { Refe- } \\
\text { rence }\end{array}$ \\
\hline \multirow[t]{2}{*}{ Bulgaria } & Burgas & 42.55 & 27.48 & A. parth. $2 \mathrm{n}(\mathrm{P})$ & F. Amat, unpubl. \\
\hline & Pomorye & 42.43 & 27.68 & $?$ & {$[27]$} \\
\hline \multirow[t]{2}{*}{ Croatia } & Secovlje, Portoroz & 45.48 & 13.60 & A. parth. 4n (P) & [27] \\
\hline & Strunjan & 45.53 & 13.60 & $?(\mathrm{P})$ & {$[27]$} \\
\hline \multirow[t]{2}{*}{ Cyprus } & Akrotiri Lake & 34.57 & 32.97 & $?$ & {$[27]$} \\
\hline & Larnaka Lake & 34.93 & 33.58 & A. salina $(\mathrm{B})$ & [27] \\
\hline \multirow[t]{17}{*}{ France } & Aigües Mortes & 43.57 & 4.18 & A. parth. 2n (P) & {$[8]$} \\
\hline & Aigües Mortes & 43.57 & 4.18 & A. franciscana $(\mathrm{B})$ & [7] \\
\hline & Carnac-Trinité sur Mer & 47.60 & -3.08 & ? & [27] \\
\hline & Guérande-le Croisic & 47.33 & -2.43 & A. parth. $4 \mathrm{n}(\mathrm{P})$ & {$[4]$} \\
\hline & Guérande-le Croisic & 47.33 & -2.43 & A. franciscana $(\mathrm{B})$ & [7] \\
\hline & La Palme & 42.83 & 3.00 & $?$ & [27] \\
\hline & Lavalduc & 43.40 & 4.93 & A. parth. $4 \mathrm{n}(\mathrm{P})$ & [4] \\
\hline & Mesquer-Assérac & 47.43 & -2.48 & $?$ & [27] \\
\hline & Porte La Nouvelle & 42.95 & 3.03 & $?$ & [27] \\
\hline & Salin de Berre & 43.40 & 5.08 & A. franciscana $(\mathrm{B})$ & {$[8]$} \\
\hline & Salin de Fos & 43.43 & 4.93 & A. franciscana $(\mathrm{B})$ & [8] \\
\hline & Salin de Giraud & 43.40 & 4.73 & A. parth. $2 \mathrm{n}(\mathrm{P})$ & [4] \\
\hline & Salin de Giraud & 43.40 & 4.73 & A. franciscana $(\mathrm{B})$ & [7] \\
\hline & Salins d'Hyères and des Pesquiers & 43.12 & 6.20 & A. franciscana $(\mathrm{B})$ & [7] \\
\hline & Sète & 43.42 & 3.70 & $?(\mathrm{P})$ & [8]; [27] \\
\hline & Sète-Villeroy & 43.38 & 3.62 & $?(\mathrm{~B})$ & [27] \\
\hline & Villeneuve & 43.53 & 3.83 & $?(\mathrm{~B})$ & [27] \\
\hline
\end{tabular}




\begin{tabular}{|c|c|c|c|c|c|}
\hline Country & Locality & $\begin{array}{l}\text { Lati- } \\
\text { tude }\end{array}$ & $\begin{array}{l}\text { Longi- } \\
\text { tude }\end{array}$ & $\begin{array}{l}\text { Species (repro- } \\
\text { ductive mode) }\end{array}$ & $\begin{array}{l}\text { Refe- } \\
\text { rence }\end{array}$ \\
\hline \multirow[t]{6}{*}{ Greece } & Citros, Pieria & 40.37 & 22.60 & A. parth. 4n $(\mathrm{P})$ & [27] \\
\hline & Kalloni & 39.17 & 26.30 & A. parth. 4n (P) & {$[27]$} \\
\hline & Megalon Embolon & 40.47 & 22.82 & A. parth. 4n (P) & {$[27]$} \\
\hline & Messolonghi & 38.30 & 21.60 & $?(\mathrm{P})$ & {$[27]$} \\
\hline & Milos & 36.58 & 24.50 & $?(\mathrm{P})$ & [27] \\
\hline & Polychnitos & 39.02 & 26.15 & A. parth. 4n $(\mathrm{P})$ & {$[27]$} \\
\hline \multirow[t]{26}{*}{ Italy } & Carloforte & 39.13 & 8.28 & A. salina $(\mathrm{B})$ & {$[27]$} \\
\hline & Cervia & 44.25 & 12.33 & A. parth. 3n (P) & [7] \\
\hline & Cervia & 44.27 & 12.35 & A. parth. 4n (P) & {$[27]$} \\
\hline & Commachio & 44.68 & 12.17 & A. parth. $4 \mathrm{n}(\mathrm{P})$ & {$[27]$} \\
\hline & Contivecchi & 39.22 & 9.03 & A. salina $(\mathrm{B})$ & {$[7]$} \\
\hline & Contivecchi & 39.22 & 9.03 & A. parth. 2n $(\mathrm{P})$ & [7] \\
\hline & Isola Longa & 37.87 & 12.43 & A. salina (B) & {$[30]$} \\
\hline & Margherita di Savoia & 41.42 & 16.08 & A. parth. 4n (P) & {$[27]$} \\
\hline & Margherita di Savoia & 41.37 & 16.08 & A. parth. $2 \mathrm{n}(\mathrm{P})$ & [7] \\
\hline & Margherita di Savoia & 41.37 & 16.08 & A. franciscana (B) & [7] \\
\hline & Mari Ermi & 39.95 & 8.40 & A. salina $(\mathrm{B})$ & {$[30]$} \\
\hline & Maria Stella & 37.98 & 12.53 & A. salina $(\mathrm{B})$ & [7] \\
\hline & Molentargius & 39.22 & 9.20 & A. parth. 2n $(\mathrm{P})$ & {$[7]$} \\
\hline & Molentargius & 39.22 & 9.20 & A. salina $(\mathrm{B})$ & [7] \\
\hline & Notteri & 39.12 & 9.52 & A. salina $(\mathrm{B})$ & {$[30]$} \\
\hline & Notteri & 39.12 & 9.52 & A. parth. $2 \mathrm{n}(\mathrm{P})$ & F. Amat, unpubl. \\
\hline & Quartu & 39.22 & 9.13 & A. salina $(\mathrm{B})$ & {$[27]$} \\
\hline & Sale Porcus & 40.02 & 8.43 & A. salina $(\mathrm{B})$ & {$[30]$} \\
\hline & Sant' Antioco & 39.03 & 8.50 & A. salina $(\mathrm{B})$ & {$[27]$} \\
\hline & Santa Gilla & 39.23 & 9.10 & A. parth. 2n (P) & {$[27]$} \\
\hline & Siracuse & 37.07 & 15.30 & $?$ & [27] \\
\hline & Su Pallosu & 40.03 & 8.40 & A. salina $(\mathrm{B})$ & {$[30]$} \\
\hline & Tarquinia & 42.48 & 11.75 & A. salina $(\mathrm{B})$ & {$[27]$} \\
\hline & Tarquinia & 41.30 & 13.33 & A. salina $(\mathrm{B})$ & {$[7]$} \\
\hline & Torre Colimena & 40.30 & 17.72 & A. parth. 2n $(\mathrm{P})$ & {$[7]$} \\
\hline & Torre Nubia & 37.97 & 12.65 & A. salina $(\mathrm{B})$ & {$[30]$} \\
\hline \multirow[t]{11}{*}{ Portugal } & Alcochete & 38.73 & -8.97 & A. franciscana $(\mathrm{B})$ & [7] \\
\hline & Aveiro, Esmolas & 40.65 & -8.68 & A. franciscana $(\mathrm{B})$ & [7] \\
\hline & Aveiro, Senitra & 40.63 & -8.65 & A. parth. 2n (P) & {$[7]$} \\
\hline & Bom Fim and Rio Frio & 38.40 & -8.57 & A. franciscana (B) & [7] \\
\hline & Castro Marim & 37.22 & -7.43 & A. franciscana $(\mathrm{B})$ & [7] \\
\hline & Olhao & 37.02 & -7.87 & A. franciscana $(\mathrm{B})$ & {$[7]$} \\
\hline & Ria de Faro & 37.03 & -7.92 & A. franciscana $(\mathrm{B})$ & {$[8]$} \\
\hline & Rio Maior & 39.35 & -8.93 & A. parth. 2n $(\mathrm{P})$ & [7] \\
\hline & Sado Estuary & 38.42 & -8.72 & A. franciscana $(\mathrm{B})$ & [8] \\
\hline & Tavira & 37.10 & -7.63 & A. franciscana $(\mathrm{B})$ & [7] \\
\hline & Tejo Estuary & 38.83 & -9.00 & A. franciscana $(\mathrm{B})$ & [7] \\
\hline
\end{tabular}




\begin{tabular}{|c|c|c|c|c|c|}
\hline Country & Locality & $\begin{array}{l}\text { Lati- } \\
\text { tude }\end{array}$ & $\begin{array}{l}\text { Longi- } \\
\text { tude }\end{array}$ & $\begin{array}{l}\text { Species (repro- } \\
\text { ductive mode) }\end{array}$ & $\begin{array}{l}\text { Refe- } \\
\text { rence }\end{array}$ \\
\hline \multirow[t]{5}{*}{ Romania } & Lacul Sârat, Brâila & 45.22 & 27.90 & $?(\mathrm{P})$ & {$[30]$} \\
\hline & Lake Techirghiol & 43.07 & 28.57 & $?(\mathrm{P})$ & {$[27]$} \\
\hline & Movila Miresii & 45.22 & 27.62 & $?$ & {$[30]$} \\
\hline & Ocna Sibiului & 45.87 & 24.05 & $?$ & [30] \\
\hline & Sovata & 46.58 & 25.08 & $?$ & {$[30]$} \\
\hline \multirow[t]{36}{*}{ Russia } & Astrakhan & 46.35 & 48.05 & $?$ & {$[30]$} \\
\hline & Bain-Tsahan & 50.25 & 115.03 & $?$ & [20] \\
\hline & Barun-Torey & 50.03 & 115.53 & $?$ & {$[20]$} \\
\hline & Baskuntchack & 48.33 & 46.92 & $?$ & [20] \\
\hline & Bolshoye Shklo & 52.58 & 79.25 & $?(\mathrm{P})$ & {$[27]$} \\
\hline & Bolshoye Yarovoye & 52.83 & 79.75 & $?(\mathrm{P})$ & [27] \\
\hline & Buazonsor & 52.67 & 79.50 & $?(\mathrm{P})$ & [27] \\
\hline & Ebeiti & 54.67 & 71.67 & $?$ & {$[20]$} \\
\hline & Elton & 49.13 & 46.67 & $?$ & [20] \\
\hline & Gorkoye & 54.17 & 77.50 & $?$ & {$[20]$} \\
\hline & Karatchi Lake & 55.33 & 76.92 & $?(\mathrm{P})$ & [20] \\
\hline & Krutoberegovoye & 54.58 & 75.67 & $?$ & {$[20]$} \\
\hline & Kuchukskoye & 52.63 & 79.50 & $?(\mathrm{P})$ & [27] \\
\hline & Kulundinskoye & 52.92 & 79.67 & $?(\mathrm{P})$ & [20] \\
\hline & Kulundinskoye & 52.92 & 79.67 & $?(\mathrm{~B})$ & [20] \\
\hline & Kurichye & 52.08 & 79.50 & $?(\mathrm{P})$ & [27] \\
\hline & Lechebnoye & 54.67 & 76.25 & $?$ & [20] \\
\hline & Malinovoye & 51.92 & 79.83 & $?(\mathrm{~B})$ & [20] \\
\hline & Maloye Yarovoye & 53.00 & 79.25 & $?(\mathrm{P})$ & [27] \\
\hline & Medvezheye & 55.30 & 67.92 & $?$ & [20] \\
\hline & Mirabilit & 52.50 & 79.08 & $?(\mathrm{P})$ & [27] \\
\hline & Mirabilit & 52.50 & 79.08 & $?(\mathrm{~B})$ & [20] \\
\hline & Mormishanskoye & 52.50 & 81.33 & $?(\mathrm{P})$ & [27] \\
\hline & Ostrovnoye & 54.33 & 75.67 & $?$ & [20] \\
\hline & Petuchovo & 52.17 & 79.50 & $?(\mathrm{P})$ & [27] \\
\hline & Schekulduk & 52.45 & 79.00 & $?(\mathrm{P})$ & {$[27]$} \\
\hline & Seledniyovo & 55.67 & 69.08 & $?(\mathrm{P})$ & {$[20]$} \\
\hline & Siverga & 55.50 & 68.67 & $?$ & {$[20]$} \\
\hline & Solyenoye & 52.75 & 81.83 & $?(\mathrm{~B})$ & {$[27]$} \\
\hline & Tanatar & 51.58 & 79.58 & $?(\mathrm{~B})$ & [27] \\
\hline & Tinaki Lake & 46.40 & 47.93 & $?$ & {$[30]$} \\
\hline & Tuchloye & 54.57 & 75.20 & $?$ & [20] \\
\hline & Tus & 54.58 & 90.08 & $?$ & [20] \\
\hline & Ulzhai & 54.33 & 75.12 & $?$ & [20] \\
\hline & Voniyucheye & 54.25 & 78.97 & $?$ & [20] \\
\hline & Zun-Torey & 50.05 & 115.75 & $?$ & [20] \\
\hline \multirow[t]{4}{*}{ Spain } & Añana & 42.80 & -2.97 & A. parth. 4n (P) & {$[30]$} \\
\hline & Arcos de las Salinas & 39.98 & -1.05 & A. parth. $4 \mathrm{n}(\mathrm{P})$ & {$[5]$} \\
\hline & Armalla & 40.90 & -1.98 & A. parth. $4 \mathrm{n}(\mathrm{P})$ & [27] \\
\hline & Ayamonte & 37.22 & -7.40 & A. parth. $2 \mathrm{n}(\mathrm{P})$ & [27] \\
\hline
\end{tabular}




\begin{tabular}{|c|c|c|c|c|c|}
\hline Country & Locality & $\begin{array}{l}\text { Lati- } \\
\text { tude }\end{array}$ & $\begin{array}{l}\text { Longi- } \\
\text { tude }\end{array}$ & $\begin{array}{l}\text { Species (repro- } \\
\text { ductive mode) }\end{array}$ & $\begin{array}{l}\text { Refe- } \\
\text { rence }\end{array}$ \\
\hline & Bonmati & 38.22 & -0.58 & A. parth. $2 \mathrm{n}(\mathrm{P})$ & {$[27]$} \\
\hline & Bonmati & 38.22 & -0.58 & A. parth. 4n (P) & {$[27]$} \\
\hline & Bonmati and Bras de Port & 38.22 & -0.58 & A. salina $(\mathrm{B})$ & {$[27]$} \\
\hline & Bras de Port & 38.22 & -0.58 & A. parth. $2 \mathrm{n}(\mathrm{P})$ & {$[5]$} \\
\hline & Burajaloz & 41.48 & -0.17 & A. parth. $4 \mathrm{n}(\mathrm{P})$ & [27] \\
\hline & Cabo de Gata & 36.80 & -2.23 & A. parth. $2 \mathrm{n}(\mathrm{P})$ & $\begin{array}{c}\text { [24]; J. Muñoz, } \\
\text { unpubl. }\end{array}$ \\
\hline & Calpe & 38.65 & 0.05 & A. parth. $2 \mathrm{n}(\mathrm{P})$ & {$[27]$} \\
\hline & Campos del Puerto, Mallorca & 39.43 & 3.02 & A. salina $(\mathrm{B})$ & {$[27]$} \\
\hline & Cerrillos Salterns & 36.68 & -2.67 & A. salina $(\mathrm{B})$ & {$[21]$} \\
\hline & Delta del Ebro & 40.57 & 0.67 & A. parth. 4n (P) & {$[7]$} \\
\hline & Delta del Ebro & 40.57 & 0.67 & A. salina $(\mathrm{B})$ & [7] \\
\hline & Delta del Ebro & 40.57 & 0.67 & A. franciscana $(\mathrm{B})$ & [7] \\
\hline & Don Benito & 37.80 & -3.72 & A. salina $(\mathrm{B})$ & {$[5]$} \\
\hline & Doñana National Park Salterns & 36.87 & -6.35 & A. salina $(\mathrm{B})$ & {$[21]$} \\
\hline & El Bosque & 36.78 & -5.55 & A. parth. 2n (P) & {$[7]$} \\
\hline & Encarnacion & 37.48 & -4.60 & A. parth. $4 \mathrm{n}(\mathrm{P})$ & [5] \\
\hline & Fuente de Piedra & 37.10 & -4.75 & A. salina $(\mathrm{B})$ & {$[5]$} \\
\hline & Fuente de Piedra & 37.10 & -4.75 & A. franciscana $(\mathrm{B})$ & [7] \\
\hline & Gerri de la Sal & 42.33 & 1.07 & A. franciscana $(\mathrm{B})$ & J. Muñoz, unpubl. \\
\hline & Gerri de la Sal & 42.33 & 1.07 & $?(\mathrm{P})$ & {$[24]$} \\
\hline & Imon & 41.17 & -2.75 & A. parth. 4n (P) & {$[27]$} \\
\hline & Isla Cristina, BIOMARIS & 37.22 & -7.32 & A. franciscana $(\mathrm{B})$ & F. Amat, unpubl. \\
\hline & Janubio, Lanzarote & 28.93 & -13.83 & A. parth. $2 \mathrm{n}(\mathrm{P})$ & {$[27]$} \\
\hline & Jumilla & 38.48 & -1.35 & A. salina $(\mathrm{B})$ & {$[5]$} \\
\hline & La Malaha & 37.10 & -3.72 & A. salina $(\mathrm{B})$ & {$[5]$} \\
\hline & La Mata & 38.03 & -0.68 & A. salina $(\mathrm{B})$ & {$[6]$} \\
\hline & La Mata & 38.03 & -0.68 & A. parth. $2 \mathrm{n}(\mathrm{P})$ & {$[30]$} \\
\hline & Laguna de Quero & 39.57 & -3.28 & $?$ & {$[24]$} \\
\hline & Lepe & 37.25 & -7.20 & A. parth. 2n (P) & {$[27]$} \\
\hline & Lerin & 42.48 & -1.98 & $?$ & [24] \\
\hline & Los Rosales Saltern & 37.88 & -4.77 & A. salina $(\mathrm{B})$ & {$[21]$} \\
\hline & Medinaceli & 41.20 & -2.50 & A. parth. $4 \mathrm{n}(\mathrm{P})$ & [27] \\
\hline & Molina del Segura & 38.05 & -1.18 & A. salina $(\mathrm{B})$ & {$[27]$} \\
\hline & Odiel & 37.25 & -6.98 & A. parth. 2n (P) & {$[7]$} \\
\hline & Odiel & 37.25 & -6.98 & A. parth. 4n (P) & {$[7]$} \\
\hline & Olmeda and Rienda & 41.10 & -2.57 & A. parth. $4 \mathrm{n}(\mathrm{P})$ & [27] \\
\hline & Peralta de la Sal & 42.00 & 0.40 & $?(\mathrm{P})$ & [27] \\
\hline & Petrola & 38.83 & -1.55 & A. parth. 4n (P) & [7] \\
\hline & Pinilla & 38.75 & -1.67 & A. parth. $4 \mathrm{n}(\mathrm{P})$ & {$[5]$} \\
\hline & Portuguesas Saltern & 36.87 & -6.33 & A. salina $(\mathrm{B})$ & {$[21]$} \\
\hline & Poza de la Sal & 42.67 & -3.50 & A. parth. $4 \mathrm{n}(\mathrm{P})$ & {$[4]$} \\
\hline & Puente Montilla & 37.52 & -4.65 & A. parth. $4 \mathrm{n}(\mathrm{P})$ & {$[5]$} \\
\hline & Puerto Real, Dolores & 36.50 & -6.15 & A. franciscana $(\mathrm{B})$ & [7] \\
\hline & Puerto Sta. Maria, La Tapa & 36.58 & -6.22 & A. franciscana (B) & [7] \\
\hline
\end{tabular}




\begin{tabular}{|c|c|c|c|c|c|}
\hline Country & Locality & $\begin{array}{l}\text { Lati- } \\
\text { tude }\end{array}$ & $\begin{array}{l}\text { Longi- } \\
\text { tude }\end{array}$ & $\begin{array}{l}\text { Species (repro- } \\
\text { ductive mode) }\end{array}$ & $\begin{array}{l}\text { Refe- } \\
\text { rence }\end{array}$ \\
\hline & Rolda & 42.17 & 0.12 & A. parth. $4 \mathrm{n}(\mathrm{P})$ & {$[5]$} \\
\hline & Sal. Catalana & 37.62 & -0.85 & A. salina $(\mathrm{B})$ & {$[24] ;[30]$} \\
\hline & Sal. Punta Galera & 37.70 & -0.90 & A. salina $(\mathrm{B})$ & {$[24] ;[30]$} \\
\hline & Salada de Chiprana & 41.23 & -0.18 & A. parth. $4 \mathrm{n}(\mathrm{P})$ & [15] \\
\hline & Salinera Española, Formentera & 38.67 & 1.43 & A. salina $(\mathrm{B})$ & [27] \\
\hline & Salinera Española, Ibiza & 38.92 & 1.58 & A. salina (B) & [27] \\
\hline & San Carlos & 37.88 & -3.67 & A. salina $(\mathrm{B})$ & {$[5]$} \\
\hline & San Felix & 36.50 & -6.33 & A. salina $(\mathrm{B})$ & [27] \\
\hline & San Fernando, El Estanquillo & 36.42 & -6.20 & A. franciscana $(\mathrm{B})$ & [7] \\
\hline & San Fernando, El Pilar & 36.48 & -6.15 & A. franciscana $(\mathrm{B})$ & [7] \\
\hline & San Juan del Puerto & 37.33 & -6.83 & A. salina $(\mathrm{B})$ & [27] \\
\hline & San Pedro del Pinatar & 37.83 & -0.83 & A. salina $(\mathrm{B})$ & {$[27]$} \\
\hline & Sanlucar de Barrameda & 36.87 & -6.32 & A. parth. $2 \mathrm{n}(\mathrm{P})$ & {$[7]$} \\
\hline & Sanlucar de Barrameda & 36.87 & -6.32 & A. salina $(\mathrm{B})$ & [7] \\
\hline & Sigüenza & 41.07 & -2.63 & ? & [24] \\
\hline & Villena & 38.65 & -0.87 & A. salina $(\mathrm{B})$ & [27] \\
\hline \multirow[t]{24}{*}{ Ukraine } & Adzhigol & 45.08 & 35.42 & $?(\mathrm{P})$ & {$[20]$} \\
\hline & Arabatskaya Strelka & 45.67 & 35.00 & $?(\mathrm{P})$ & [20] \\
\hline & Bolshoye Otra Moynakskyoe & 45.00 & 33.00 & $?$ & [27] \\
\hline & Dolgoye & 46.50 & 35.58 & $?(\mathrm{P})$ & [20] \\
\hline & Dzharylhatch & 45.57 & 30.57 & $?(\mathrm{P})$ & {$[20]$} \\
\hline & Ghenicheskoye Lake & 46.25 & 34.67 & $?$ & [27] \\
\hline & Kinburgski peninsula & 46.42 & 31.67 & $?$ & {$[20]$} \\
\hline & Kizil-Yar & 45.05 & 33.92 & $?(\mathrm{P})$ & [20] \\
\hline & Kizil-Yar & 45.05 & 33.92 & A. salina $(\mathrm{B})$ & [20] \\
\hline & Kujalnicsky Liman & 46.72 & 30.58 & A. parth. $2 \mathrm{n}(\mathrm{P})$ & $\begin{array}{l}\text { [20]; J. Muñoz, } \\
\text { unpubl. }\end{array}$ \\
\hline & Odzhigol & 46.42 & 32.22 & $?$ & {$[20]$} \\
\hline & Otar-Moinakskoye & 45.20 & 33.50 & $?(\mathrm{P})$ & [20] \\
\hline & Popovskoye & 45.28 & 33.08 & $?(\mathrm{P})$ & [20] \\
\hline & Popovskoye & 45.28 & 33.08 & A. salina $(\mathrm{B})$ & [20] \\
\hline & Sakshoye Lake & 45.17 & 33.50 & $?(\mathrm{P})$ & [20] \\
\hline & Sasik-Sivash & 44.83 & 33.42 & $?(\mathrm{P})$ & [20] \\
\hline & Sasik-Sivash & 44.83 & 33.42 & A. salina $(\mathrm{B})$ & [20] \\
\hline & Shtormovoye & 45.33 & 53.08 & A. salina (B) & [20] \\
\hline & Sivashskoye & 46.38 & 34.55 & $?(\mathrm{~B})$ & [30] \\
\hline & Sredneye & 46.50 & 35.50 & $?(\mathrm{P})$ & [20] \\
\hline & Tchokrakskoye & 45.42 & 36.25 & $?(\mathrm{~B})$ & [9] \\
\hline & Tchokrakskoye & 45.42 & 36.25 & $?(\mathrm{P})$ & [20] \\
\hline & Tchongar & 46.00 & 34.58 & $?(\mathrm{P})$ & [20] \\
\hline & Tobechikskoye & 45.17 & 36.33 & $?(\mathrm{P})$ & [27] \\
\hline Montenegro & Ulcinj & 41.92 & 19.20 & $?(\mathrm{P})$ & [27] \\
\hline
\end{tabular}




\section{NORTH AMERICA}

\begin{tabular}{|c|c|c|c|c|c|}
\hline Country & Locality & $\begin{array}{l}\text { Lati- } \\
\text { tude }\end{array}$ & $\begin{array}{l}\text { Longi- } \\
\text { tude }\end{array}$ & $\begin{array}{l}\text { Species (repro- } \\
\text { ductive mode) }\end{array}$ & $\begin{array}{l}\text { Refe- } \\
\text { rence }\end{array}$ \\
\hline \multirow[t]{38}{*}{ Canada } & Akerlund Lake & 52.30 & -109.25 & $?(\mathrm{~B})$ & {$[27]$} \\
\hline & Alsask Lake & 51.33 & -109.87 & $?(\mathrm{~B})$ & {$[27]$} \\
\hline & Aroma Lake & 51.30 & -108.55 & $?(\mathrm{~B})$ & [27] \\
\hline & Berry Lake & 52.12 & -105.50 & $?(\mathrm{~B})$ & [27] \\
\hline & Boat Lake & 50.28 & -109.98 & $?(\mathrm{~B})$ & [27] \\
\hline & Burn Lake & 49.82 & -105.45 & $?(\mathrm{~B})$ & [27] \\
\hline & Ceylon Lake & 49.45 & -104.60 & $?(\mathrm{~B})$ & {$[27]$} \\
\hline & Chain Lake & 50.50 & -108.72 & $?(\mathrm{~B})$ & [27] \\
\hline & Chaplin Lake & 50.42 & -106.63 & A. franciscana $(\mathrm{B})$ & {$[27]$} \\
\hline & Churchill & 58.75 & -94.00 & $?(\mathrm{~B})$ & [27] \\
\hline & Coral Lake & 49.85 & -102.35 & $?(\mathrm{~B})$ & {$[27]$} \\
\hline & Drybore Lake & 49.72 & -105.50 & $?(\mathrm{~B})$ & {$[27]$} \\
\hline & Enis Lake & 52.17 & -108.32 & $?(\mathrm{~B})$ & [27] \\
\hline & Frederick Lake & 49.98 & -105.63 & $?(\mathrm{~B})$ & [27] \\
\hline & Fusilier Lake & 51.83 & -109.73 & $?(\mathrm{~B})$ & [27] \\
\hline & Grandora Lake & 52.10 & -107.00 & $?(\mathrm{~B})$ & [27] \\
\hline & Gull Lake & 50.10 & -108.45 & $?(\mathrm{~B})$ & {$[27]$} \\
\hline & Hatton Lake & 50.03 & -109.83 & $?(\mathrm{~B})$ & [27] \\
\hline & Horizon Lake & 49.53 & -105.28 & $?(\mathrm{~B})$ & [27] \\
\hline & Ingebright Lake & 50.37 & -109.32 & $?(\mathrm{~B})$ & [27] \\
\hline & La Perouse & 55.23 & -98.00 & $?(\mathrm{~B})$ & [27] \\
\hline & Landis Lake & 52.22 & -108.45 & $?(\mathrm{~B})$ & [27] \\
\hline & Little Manitou Lake & 51.80 & -105.50 & A. franciscana (B) & [27] \\
\hline & Lydden Lake & 52.15 & -108.22 & $?(\mathrm{~B})$ & [27] \\
\hline & Mawer Lake & 50.77 & -106.37 & $?(\mathrm{~B})$ & [27] \\
\hline & Meacham Lake & 52.12 & -105.78 & $?(\mathrm{~B})$ & [27] \\
\hline & Muskiki Lake & 52.33 & -105.75 & $?(\mathrm{~B})$ & [27] \\
\hline & Neola Lake & 52.05 & -107.82 & $?(\mathrm{~B})$ & {$[27]$} \\
\hline & Oban Lake & 52.15 & -108.15 & $?(\mathrm{~B})$ & [27] \\
\hline & Richmond Lake & 52.02 & -108.02 & $?(\mathrm{~B})$ & [27] \\
\hline & Shoe Lake & 49.92 & -105.45 & $?(\mathrm{~B})$ & [27] \\
\hline & Snakehole Lake & 50.50 & -108.50 & $?(\mathrm{~B})$ & [27] \\
\hline & Sybouts Lake-East & 49.03 & -104.40 & $?(\mathrm{~B})$ & [27] \\
\hline & Sybouts Lake-West & 49.03 & -104.45 & $?(\mathrm{~B})$ & [27] \\
\hline & Verlo West & 50.32 & -108.62 & $?(\mathrm{~B})$ & [27] \\
\hline & Vincent Lake & 50.22 & -108.95 & $?(\mathrm{~B})$ & [27] \\
\hline & Wheatsone Lake & 49.82 & -105.40 & $?(\mathrm{~B})$ & [27] \\
\hline & Whiteshore Lake & 52.13 & -108.28 & $?(\mathrm{~B})$ & [27] \\
\hline \multicolumn{6}{|l|}{ USA } \\
\hline Arizona & Kiatuthlana Green and Red Pond & 34.83 & -109.43 & A. franciscana $(\mathrm{B})$ & {$[27]$} \\
\hline
\end{tabular}




\begin{tabular}{|c|c|c|c|c|c|}
\hline Country & Locality & $\begin{array}{l}\text { Lati- } \\
\text { tude }\end{array}$ & $\begin{array}{l}\text { Longi- } \\
\text { tude }\end{array}$ & $\begin{array}{l}\text { Species (repro- } \\
\text { ductive mode) }\end{array}$ & $\begin{array}{l}\text { Refe- } \\
\text { rence }\end{array}$ \\
\hline \multirow[t]{9}{*}{ California } & Carpineteria Slough & 34.40 & -119.50 & $?(\mathrm{~B})$ & [27] \\
\hline & Chula Vista & 32.60 & -117.08 & $?(\mathrm{~B})$ & [27] \\
\hline & Mono Lake & 38.00 & -119.00 & A. monica $(\mathrm{B})$ & [27] \\
\hline & Moss Landing, Monterey Bay & 36.80 & -121.77 & A. franciscana $(\mathrm{B})$ & [27] \\
\hline & Owens Lake & 36.42 & -117.93 & ? (B) & [27] \\
\hline & San Diego & 32.83 & -117.17 & $?(\mathrm{~B})$ & [27] \\
\hline & San Francisco Bay & 37.47 & -122.50 & A. franciscana $(\mathrm{B})$ & [27] \\
\hline & San Pablo Bay & 38.00 & -122.27 & A. franciscana $(\mathrm{B})$ & [27] \\
\hline & Vallejo West Pond & 38.20 & -122.25 & $?(\mathrm{~B})$ & [27] \\
\hline \multirow[t]{3}{*}{ Hawaii } & Christmas Islands & 1.83 & -157.33 & $?(\mathrm{~B})$ & [27] \\
\hline & Hanapepe & 21.90 & -159.50 & $?(\mathrm{~B})$ & [27] \\
\hline & Laysan Atoll & 25.50 & -167.00 & A. franciscana $(\mathrm{B})$ & [27] \\
\hline \multirow[t]{5}{*}{ Nebraska } & Alkali Lake & 43.53 & -100.63 & $?(\mathrm{~B})$ & [27] \\
\hline & Antioch (Potash) Lake & 42.07 & -102.57 & A. franciscana $(\mathrm{B})$ & [27] \\
\hline & Ashenburger Lake Complex & 42.00 & -102.00 & $?(\mathrm{~B})$ & [27] \\
\hline & Jesse Lake & 42.10 & -102.65 & A. franciscana $(\mathrm{B})$ & [27] \\
\hline & Sturgeon Lake & 41.98 & -102.67 & A. franciscana $(\mathrm{B})$ & [27] \\
\hline Nevada & Fallon Pond & 39.52 & -118.87 & A. franciscana $(\mathrm{B})$ & [27] \\
\hline \multirow[t]{2}{*}{ North Dakota } & Miller Lake & 48.83 & -103.95 & $?(\mathrm{~B})$ & [29] \\
\hline & Stink (Williams) Lake & 48.63 & -104.00 & $?(\mathrm{~B})$ & [29] \\
\hline \multirow[t]{4}{*}{ New Mexico } & Laguna del Perro & 34.53 & -106.02 & $?(\mathrm{~B})$ & [27] \\
\hline & Loving Salt Lake & 32.28 & -104.07 & $?(\mathrm{~B})$ & [27] \\
\hline & Quemado & 34.28 & -108.47 & A. franciscana $(\mathrm{B})$ & [27] \\
\hline & Zuni Salt Lake & 34.45 & -108.77 & A. franciscana (B) & [27] \\
\hline Oregon & Lake Abert & 42.58 & -120.25 & $?(\mathrm{~B})$ & [27] \\
\hline \multirow[t]{7}{*}{ Texas } & Cedar Lake & 34.80 & -102.27 & A. franciscana $(\mathrm{B})$ & [27] \\
\hline & McKenzies Playa & 32.68 & -102.17 & $?(\mathrm{~B})$ & [27] \\
\hline & Mound Playa & 33.17 & -101.93 & ? (B) & [27] \\
\hline & Playa Thahoka & 33.20 & -101.57 & $?(\mathrm{~B})$ & [27] \\
\hline & Raymondville & 26.17 & -97.80 & ? (B) & [27] \\
\hline & Rich Playa & 33.22 & -102.05 & $?(\mathrm{~B})$ & [27] \\
\hline & Snow drop Playa & 32.98 & -101.67 & $?(\mathrm{~B})$ & [27] \\
\hline Utah & Great Salt Lake & 41.00 & -112.50 & A. franciscana (B) & [27] \\
\hline \multirow[t]{6}{*}{ Washington } & Cameron Lake & 48.30 & -119.53 & A. franciscana $(\mathrm{B})$ & [27] \\
\hline & Deposit Thirteen & 48.22 & -119.50 & A. franciscana $(\mathrm{B})$ & [27] \\
\hline & Hot (Bitter) Lake & 48.97 & -119.48 & A. franciscana (B) & [27] \\
\hline & Omak Plateau & 48.42 & -119.40 & $?(\mathrm{~B})$ & [27] \\
\hline & Penley Lake & 48.28 & -119.53 & A. franciscana $(\mathrm{B})$ & [27] \\
\hline & Soap Lake & 47.55 & -119.42 & $?(\mathrm{~B})$ & [27] \\
\hline
\end{tabular}




\section{Supplementary Map}

Native area

Invaded area

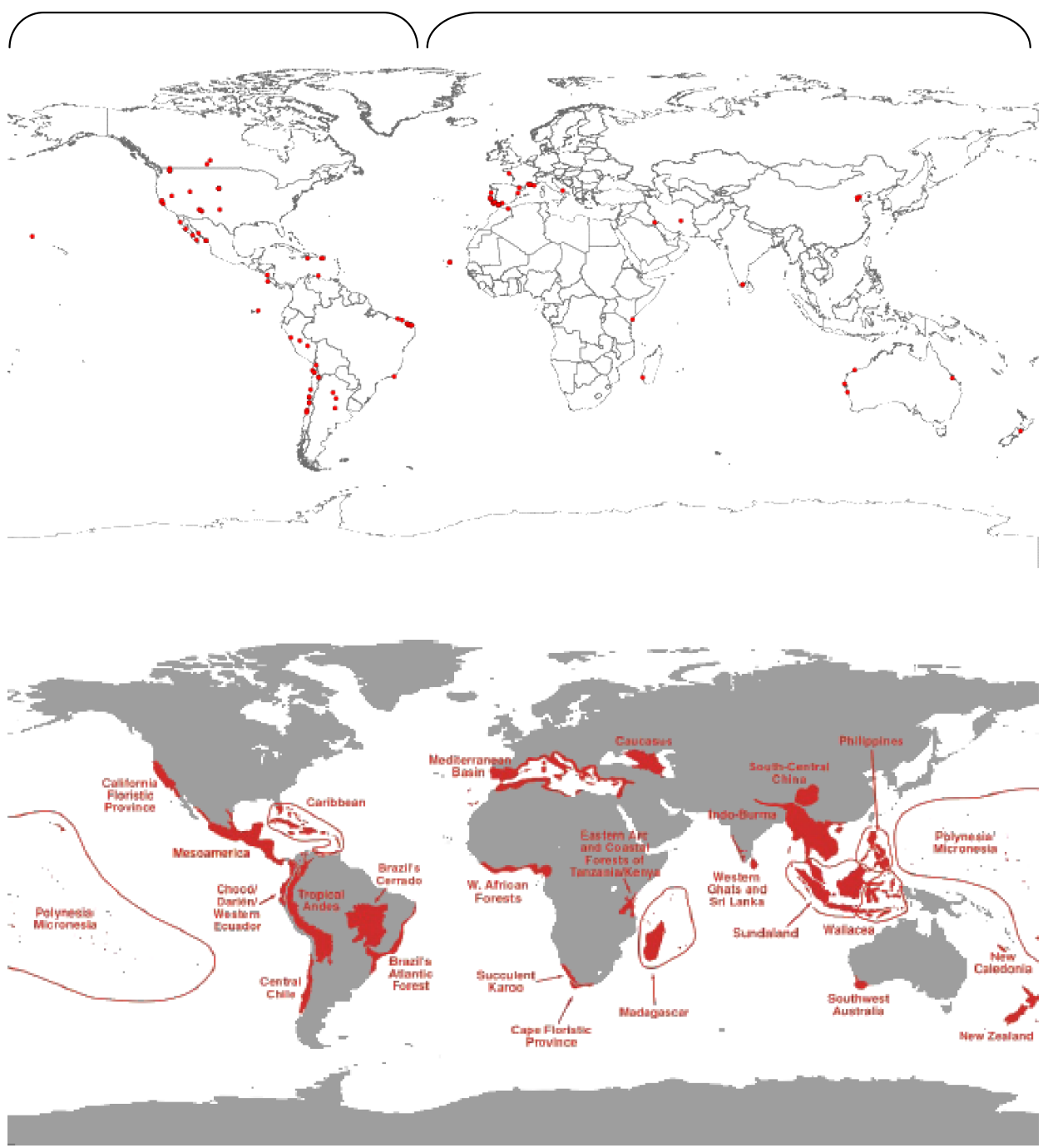

Fig. 3. Distribution of Artemia franciscana Kellogg, 1906 localities (red circles) around the world, indicating native and invasive areas (upper map) comparing the identified hotspots in the exact map as reported by Myers et al. (2000) (lower map). 


\section{REFERENCES}

[1] Abatzopoulos, T. J., N. Agh, G. Van Stappen, S. M. Razavi Rouhani \& P. SorGELOOS, 2006. Artemia sites in Iran. Journal of the Marine Biological Association of the United Kingdom, 86: 299-307.

[2] Abatzopoulos, T. J., I. Kappas, P. Bossier, P. Sorgeloos \& J. A. Beardmore, 2002. Genetic characterization of Artemia tibetiana (Crustacea: Anostraca). Biological Journal of the Linnean Society, 75: 333-344.

[3] Амat, F., 1979. Diferenciación y distribución de las poblaciones de Artemia (Crustáceo, Branquiópodo) de España: 1-250. (Ph.D. Thesis, Universidad de Barcelona).

[4] Aмat, F., 1983. Diferenciación y distribución de las poblaciones de Artemia en España. VI. Biogeografía. Investigación Pesquera, Barcelona, 47(2): 231-240.

[5] Amat, F., C. Barata, F. Hontoria, J. C. NAVArRo \& I. VAró, 1995. Biogeography of the genus Artemia (Crustacea, Branchiopoda, Anostraca) in Spain. International Journal of Salt Lake Research, 3: 175-190.

[6] Amat, F., F. Hontoria, J. C. Navarro, A. Gonzalbo \& I. Varó, 1991. Bioecología de Artemia (Crustacea, Branchiopoda) en la laguna de La Mata (Torrevieja, Alicante). Instituto de Estudios 'Juan Gil Albert': 1-173. (Diput. Provincial de Alicante, Spain).

[7] Amat, F., F. Hontoria, J. C. Navarro, N. Vieira \& G. Mura, 2007. Biodiversity loss in the genus Artemia in the Western Mediterranean Region. Limnetica, 26(2): 177-194.

[8] Amat, F., F. Hontoria, O. Ruiz, A. J. Green, M. I. Sánchez, J. Figuerola \& F. HoRTAS, 2005. The American brine shrimp as an exotic invasive species in the Western Mediterranean. Biological Invasions, 7: 37-47.

[9] Baitchorov, V. M. \& L. L. NAgOrskaya, 1999. The reproductive characteristics of Artemia in habitats of different salinity. International Journal of Salt Lake Research, 8: 287-291.

[10] Basbug, Y. \& F. Y. Demirkalp, 1997. A note on the brine shrimp Artemia in Tuz Lake (Turkey). Hydrobiologia, 353: 45-51.

[11] Camara, M. R., 2001. Dispersal of Artemia franciscana Kellogg (Crustacea; Anostraca) populations in the coastal saltworks of Rio Grande do Norte, northeastern Brazil. Hydrobiologia, 466: 145-148.

[12] Campos, H., D. Soto, O. Parra, W. Steffen \& G. Agüero, 1996. Limnological studies of Amarga Lagoon, Chile: a saline lake in Patagonian South America. International Journal of Salt Lake Research, 4: 301-314.

[13] Cohen, R. G., F. Amat, F. Hontoria \& J. C. NAVArro, 1999. Preliminary characterization of some Argentinean Artemia populations from La Pampa and Buenos Aires provinces. International Journal of Salt Lake Research, 8: 329-340.

[14] De Los Rios, P. R. \& O. ZuÑIGA, 2000. Comparación biométrica del lóbulo frontal en poblaciones americanas de Artemia (Anostraca, Artemiidae). Revista Chilena de Historia Natural, 73(1): 31-38. Available on website http://www.scielo.cl/scielo.php?script=sci_ arttext\&pid=S0716-078X2000000100004\&lng=es\&nrm=iso

[15] Gómez, A., M. Serra, G. R. Carvalho \& D. H. Lunt, 2002. Speciation in ancient cryptic species complexes: evidence from the molecular phylogeny of Brachionus plicatillis (Rotifera). Evolution, 56(7): 1431-1444.

[16] Kaiser, H., A. K. Gordon \& T. G. Paulet, 2006. Review of the African distribution of the brine shrimp genus Artemia. Water SA, 32(4): 597-604. Available on website http://www.wrc.org.za

[17] MAYER, R. J., 2002. Morphology and biometry of three populations of Artemia (Branchiopoda: Anostraca) from the Dominican Republic and Puerto Rico. Hydrobiologia, 486: 29-38. 
[18] Mcmaster, K., A. Savage, T. Finston, M. S. Johnson \& B. Knott, 2007. The recent spread of Artemia parthenogenetica in Western Australia. Hydrobiologia, 576: 39-48.

[19] Mirabdullayev, I. M., I. M. Joldarova, Z. A. Mustafaeva, S. Kazakhbaev, S. A. Lyubimova \& B. A. Tashmukhamedov, 2004. Succession of the ecosystems of the Aral Sea during its transition from oligohaline to polyhaline water body. Journal of Marine Systems, 47(1-4): 101-107.

[20] Mura, G. \& L. NAgorskaya, 2005. Notes on the distribution of the genus Artemia in the former USSR countries (Russia and adjacent regions). Journal of Biological Research, 4: $139-150$.

[21] Muñoz, J., A. Gómez, A. J. Green, J. Figuerola, F. Amat \& C. Rico, 2008. Phylogeography and local endemism of the native Mediterranean brine shrimp Artemia salina (Branchiopoda: Anostraca). Molecular Ecology, 17: 3160-3177.

[22] OdIo, R., 1991. Artemia in Costa rica. Larviculture and Artemia Newsletter, 19: 39.

[23] Ruiz, O., G. R. Medina, R. G. Cohen, F. Amat \& J. C. Navarro, 2007. Diversity of the fatty acid composition of Artemia spp. cysts from Argentinean populations. Marine Ecology Progress Series, 335: 155-165.

[24] Sorgeloos, P., P. LAVEns, P. LÈ, W. Tackaert \& D. Versichele, 1986. Manual para el cultivo y uso de Artemia en acuicultura. PROGRAMA COOPERATIVO GUBERNAMENTAL FAO - ITALIA. GCP/RLA/075/ITA. Documento de Campo 10. Belgium Available on website http://www.fao.org/docrep/field/003/AB474S/AB474S00.HTM.

[25] Torrentera, L. \& S. I. Dodson, 2004. Ecology of the brine shrimp Artemia in the Yucatan, Mexico, Salterns. Journal of Plankton Research, 26(6): 617-624.

[26] Triantaphyllidis, G. V., T. J. Abatzopoulos, E. Miasa \& P. Sorgeloos, 1996. International study on Artemia. LVI. Characterization of two Artemia populations from Namibia and Madagascar: cytogenetics, biometry, hatching characteristics and fatty acid profiles. Hydrobiologia, 335: 97-106.

[27] Triantaphyllidis, G. V., T. J. Abatzopoulos \& P. Sorgeloos, 1998. Review of the biogeography of the genus Artemia (Crustacea, Anostraca). Journal of Biogeography, 25: 213-226.

[28] Vallell, L. L., 2006. Caracterización de las poblaciones de Artemia presentes en Cabo Verde. Tesina de Master en Acuicultura: 1-50. (Universidad de Las Palmas de Gran Canaria, Spain).

[29] VAnhaecke, P., W. TACKaert \& P. Sorgeloos, 1987. The biogeography of Artemia: an updated review. In: P. Sorgeloos, D. A. Bengtson, W. DeCleir \& E. Jaspers (eds.), Artemia research and its applications: 129-155. (Universa Press, Wetteren).

[30] Van Stappen, G., 2002. Chapter IV: Zoogeography. In: T. J. Abatzopoulos, J. A. Beardmore, J. S. Clegg \& P. Sorgeloos (eds.), Biology of aquatic organisms. Artemia: basic and applied biology: 171-224. (Kluwer Academic Publishers).

[31] Van Stappen, G., H. Yu, X. Wang, S. Hoffman, K. Cooreman., P. Bossier \& P. SORGELOOS, 2007. Occurence of allochthonous Artemia species in the Bohai Bay area, PR China, as confirmed by RFLP analysis and laboratory culture tests. Fundamental and Applied Limnology, 170(1): 21-28.

[32] Xin, N., J. Sun, B. Zhang, G. V. Triantaphyllidis, G. Van Stappen \& P. SorGELOOS, 1994. International study on Artemia*. LI. New survey of Artemia resources in the People's Republic of China. International Journal of Salt Lake Research, 3: 105-112.

[33] Zuñiga, O., R. Wilson, F. Amat \& F. Hontoria, 1999. Distribution and characterization of Chilean populations of the brine shrimp Artemia (Crustacea, Branchiopoda, Anostraca) International Journal of Salt Lake Research, 8: 23-40.

Updates for the data here presented can be found at: http://www.ebd.csic.es/quini/ QuiniPersonal_E.htm 